\title{
Steady rimming flows with surface tension
}

\author{
E. S. BENILOV ${ }^{1}$, M. S. BENILOV \\ AND N. KOPTEVA ${ }^{1}$ \\ ${ }^{1}$ Department of Mathematics, University of Limerick, Ireland \\ ${ }^{2}$ Department of Physics, University of Madeira, Portugal
}

(Received 8 March 2006 and in revised form 10 October 2007)

We examine steady flows of a thin film of viscous fluid on the inside of a cylinder with horizontal axis, rotating about this axis. If the amount of fluid in the cylinder is sufficiently small, all of it is entrained by rotation and the film is distributed more or less evenly. For medium amounts, the fluid accumulates on the 'rising' side of the cylinder and, for large ones, pools at the cylinder's bottom. The paper examines rimming flows with a pool affected by weak surface tension. Using the lubrication approximation and the method of matched asymptotics, we find a solution describing the pool, the 'outer' region, and two transitional regions, one of which includes a variable (depending on the small parameter) number of asymptotic zones.

\section{Introduction}

Rimming flows, i.e. flows of a viscous fluid on the inside of a rotating horizontal cylinder, have important industrial applications and are also of great interest to theoreticians. The case when the fluid layer is sufficiently thin and is fully entrained by the cylinder's rotation has been examined by Moffatt (1977), who found a family of asymptotic solutions describing a steady-state distribution of liquid film on the cylinder's surface. If, however, the net mass $M$ of the fluid inside the cylinder exceeds a certain threshold, $M_{*}$, the film is no longer thin enough for viscous entrainment to 'overcome' gravity. As a result, fluid parcels cannot climb past the point where the tangent to the cylinder's surface is vertical and the effect of gravity is, thus, the strongest. These parcels accumulate on the rising side of the cylinder, where the film becomes so thick, that the fluid starts falling back and forms a 'shock' (Benjamin, Pritchard \& Tavener 1993, O'Brien \& Gath 1998) similar to that of a hydraulic jump or a tidal bore.

It turns out, however, that the net mass of a shock solution may not exceed a certain threshold value, $M_{* *}$. Physically, this means that the cylinder's rising side can accommodate no more than a certain amount of fluid, with the excess fluid pooling at the bottom. As shown by Ashmore, Hosoi \& Stone (2003), the 'pool' can be strongly affected by surface tension, but (as we shall see in $\S 7$ ) their results are applicable only if $M \gg M_{* *}$.

The present paper re-examines the setting considered by Ashmore et al. (2003), i.e. a steady rimming flow affected by weak surface tension, for $M \gtrsim M_{* *}$. In $\S 2$, we formulate the problem and, in $\S \S 3-4,6$, solve it asymptotically. In $\S 5$, asymptotic results are verified and complemented numerically. 


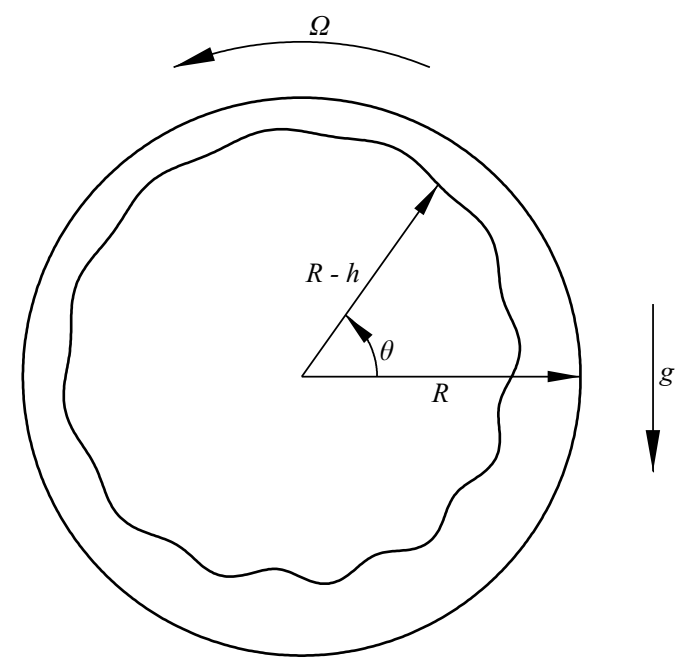

FIGURE 1. Liquid film in a rotating horizontal cylinder.

\section{Formulation of the problem}

Consider a thin liquid film on the inside of a cylinder of radius $R$ with horizontal axis, rotating about this axis with a constant angular velocity $\Omega$ (see figure 1 ). We are concerned with two-dimensional flows, described by polar coordinates $\left(r_{*}, \theta_{*}\right)$, so the thickness $h_{*}$ of the film depends on the azimuthal angle $\theta_{*}$ and time $t_{*}$ (asterisks denote dimensional variables). We shall also introduce the acceleration due to gravity, $g$, the fluid's density $\rho$, kinematic viscosity $\nu$, and surface tension $\sigma$.

In what follows, we use the following non-dimensional variables:

$$
h=\frac{h_{*}}{\alpha R}, \quad \theta=\theta_{*}, \quad t=\Omega t_{*},
$$

where

$$
\alpha=\left(\frac{\nu \Omega}{g R}\right)^{1 / 2} .
$$

We also introduce a non-dimensional parameter characterising surface tension,

$$
\epsilon=\frac{\sigma}{\rho g R^{2}}\left(\frac{\nu \Omega}{g R}\right)^{1 / 2} .
$$

\subsection{The governing equations}

Following most studies of rimming flows, we employ the so-called lubrication theory, assuming the film to be thin and the slope of its surface small - resulting in the following evolution equation:

$$
\frac{\partial h}{\partial t}+\frac{\partial}{\partial \theta}\left[h-\frac{1}{3} h^{3} \cos \theta+\frac{1}{3} \epsilon h^{3}\left(\frac{\partial h}{\partial \theta}+\frac{\partial^{3} h}{\partial \theta^{3}}\right)\right]=0
$$

(Johnson 1990). In equation (2.4), the first term in square brackets describes viscous entrainment of the film by the rotation of the cylinder, the second term describes the effect of gravity, and the term involving $\epsilon$, surface tension. 
We are concerned with steady-state solutions, $h=h(\theta)$, in which case equation (2.4) yields

$$
h-\frac{1}{3} h^{3} \cos \theta+\frac{1}{3} \epsilon h^{3}\left(\frac{\mathrm{d} h}{\mathrm{~d} \theta}+\frac{\mathrm{d}^{3} h}{\mathrm{~d} \theta^{3}}\right)=q,
$$

where the constant of integration $q$ is, physically, the non-dimensional flux. Equation (2.5) should be supplemented by the periodicity condition,

$$
h(\theta+2 \pi)=h(\theta) .
$$

Problem (2.5)-(2.6) describes a family of solutions with various values of $q$. It is more convenient, however, to characterize the solutions by the non-dimensional net mass $M$, i.e. impose an additional constraint,

$$
\int_{0}^{2 \pi} h \mathrm{~d} \theta=M
$$

The flux in this case should be treated as a function of the mass, $q(M)$, to be determined from (2.5)-(2.7).

Following all previous work on this and similar problems, we shall assume

$$
\epsilon \ll 1 \text {. }
$$

\subsection{The leading-order results $(\epsilon=0)$}

To leading order, equation (2.5) becomes

$$
h-\frac{1}{3} h^{3} \cos \theta=q .
$$

This cubic equation was examined by Moffatt (1977), who demonstrated that, if $q<\frac{2}{3}$, (2.8) has a smooth unique solution (see figure $2 a$ ). For $q=\frac{2}{3}, h(\theta)$ has a 'corner' at $\theta=0$ (see figure $2 b$ ); note also that the net mass of this solution is

$$
M_{*} \approx 4.44,
$$

as computed numerically. Recall that $M_{*}$ is the threshold separating continuous and shock solutions.

In order to understand the nature of the shock solutions, note that, in the first and fourth quadrants, equation (2.8) has two positive roots, with the smaller of the two corresponding to continuous solutions (see the left-hand panels of figure 2). For $q=\frac{2}{3}$, the two roots touch at $\theta=0$ and allow a continuous transition from the smaller root to the larger one (see the left-hand panels of figure 3 ). Observe that, as $\theta \rightarrow \frac{1}{2} \pi, \frac{3}{2} \pi$, the larger root becomes infinite - hence, the solution must 'jump' back to the smaller root before that. As a result, two types of solutions exist (Benjamin et al. 1993; O’Brien \& Gath 1998), with shocks in the first and fourth quadrants - see figures $3(b)$ and $(a)$ respectively. The former, however, is unstable (Benjamin et al. 1993; O'Brien 2002), making the latter the only meaningful shock solution. $\dagger$

Note that all shock solutions correspond to the same value of flux, $q=\frac{2}{3}$, i.e. there exists a limiting amount of fluid per unit time which can be transferred across the roof of the cylinder. The excess fluid accumulates on the cylinder's rising side, between

$\dagger$ In 'real' rimming flows, shocks have never been observed, as they are always smoothed by surface tension and/or a breakdown of lubrication theory due to large slopes of the film's surface. In the latter case, a cusp can form at the 'foot' of the shock, similar to that examined by Jeong \& Moffatt (1992). 

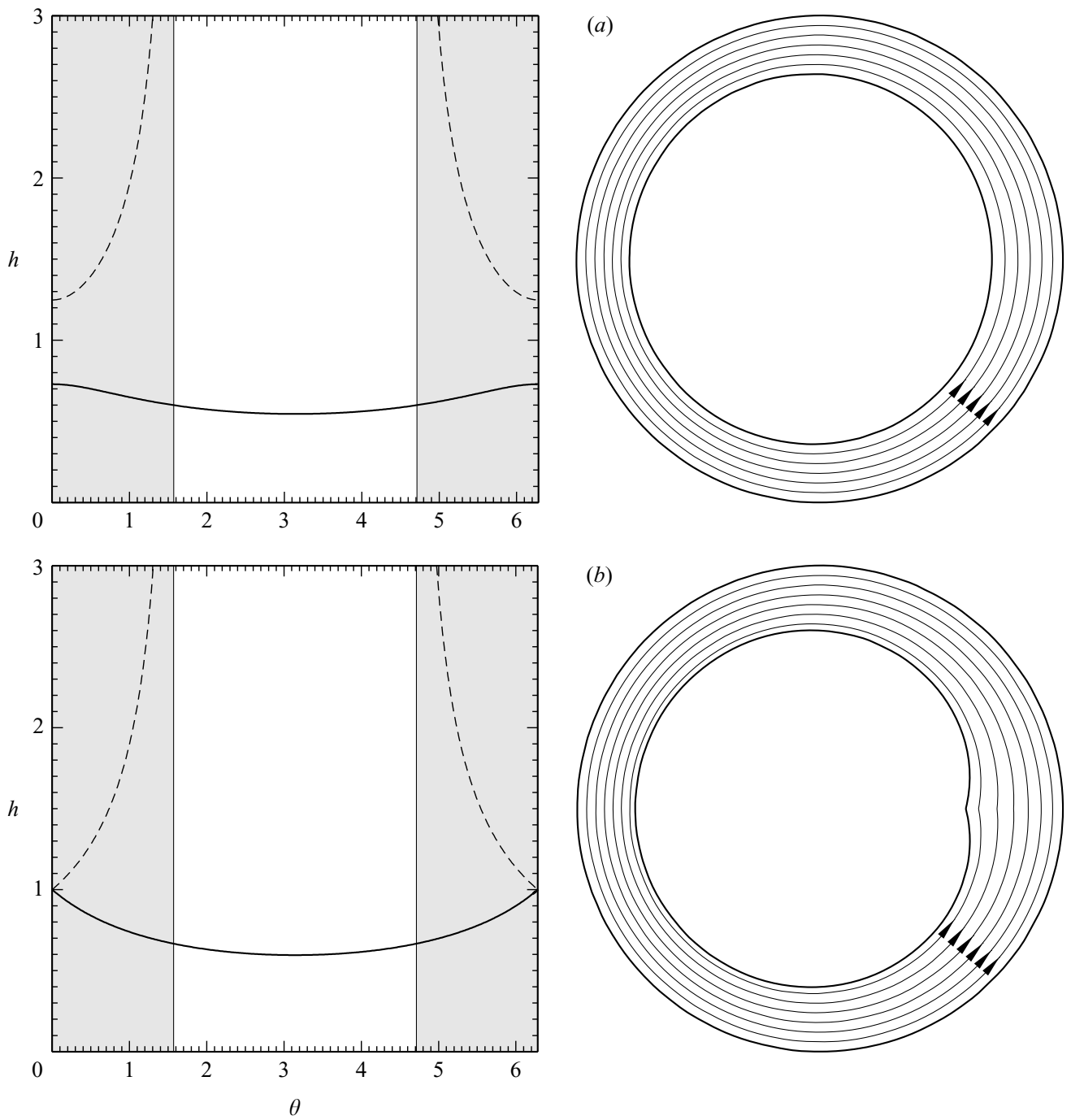

FIGURE 2. Rimming flows with continuous profile: (a) $q=0.6 ;$ (b) $q=\frac{2}{3}$. The left-hand panels show the non-dimensional solution $h(\theta)$ : the solid/dashed line corresponds to the smaller/larger root of equation (2.8), the first and fourth quadrants are shaded. The right-hand panels show the 'real' solution, the scale of which implies $\alpha=0.4$, where $\alpha$ is given by (2.2) (the relatively large value of $\alpha$ has been chosen to improve visibility of the fine structure of the flow).

the shock and the point $\theta=0$, which seems to suggest that the latter is a stagnation point. To clarify this issue, we computed the streamlines (see Appendix A), according to which $\theta=0$ is not a stagnation point - see figure 3 .

To resolve the paradox, consider the 'local' flux

$$
Q(h, \theta)=h-\frac{1}{3} h^{3} \cos \theta,
$$

which is, essentially, the left-hand side of equation (2.8). For points on the rising side of the cylinder, i.e. for $\theta \in\left(-\frac{1}{2} \pi, \frac{1}{2} \pi\right)$, this expression has a maximum at $h=(\cos \theta)^{-1 / 2}$, 

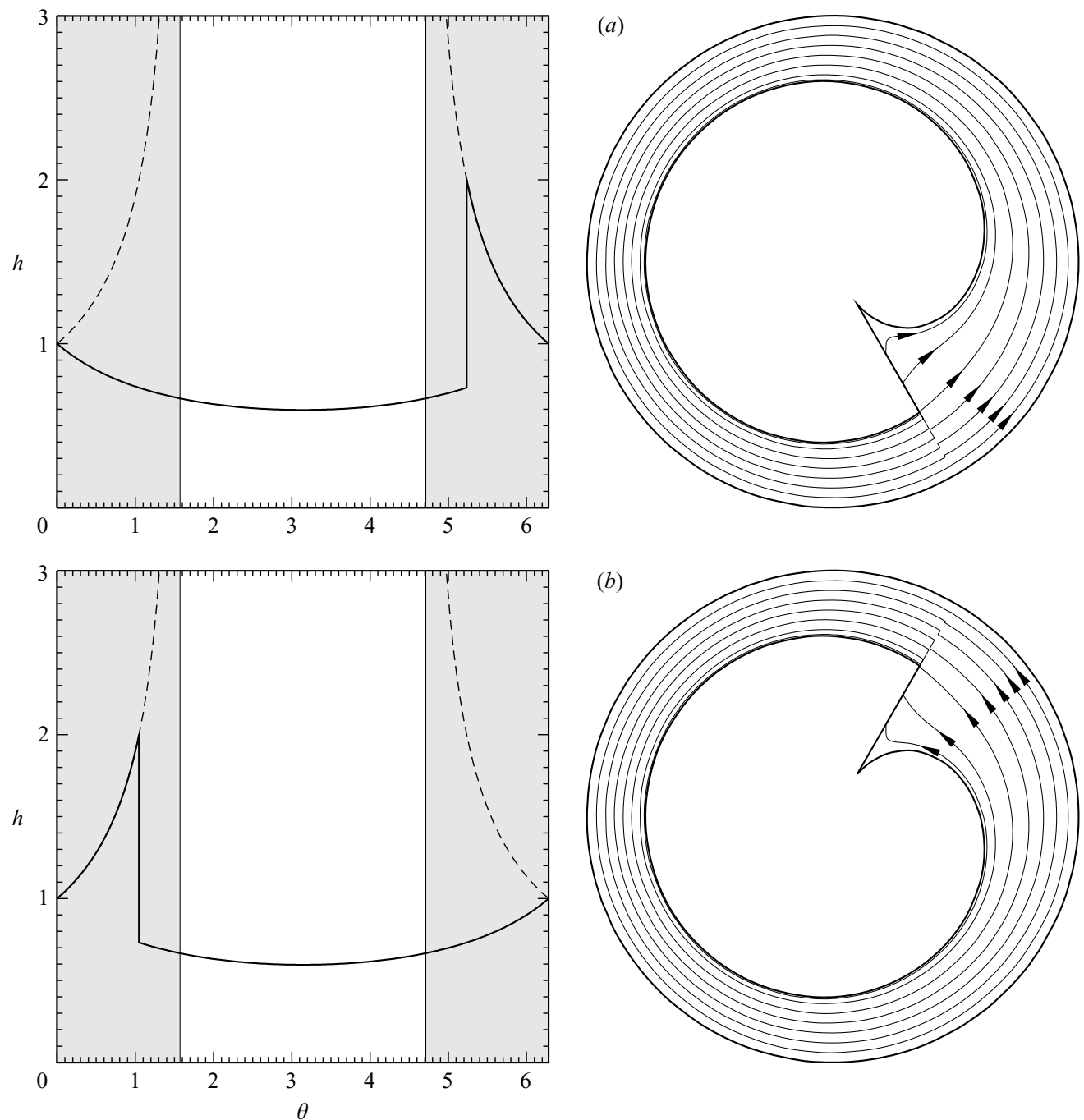

FIGURE 3. Rimming flows with shocks (as in figure 2, the spatial scale of the right-hand panels corresponds to $\alpha=0.4$ ). (a) The shock is located at $\theta=\frac{5}{3} \pi$, in the fourth quadrant (stable solution); (b) the shock is located at $\theta=\frac{1}{3} \pi$, in the first quadrant (unstable solution).

such that

$$
Q_{\max }=\frac{2}{3}(\cos \theta)^{-1 / 2} .
$$

Clearly, the maximum global flux may not exceed the local flux at the most unfavourable point, i.e. where $\cos \theta=1$ and $Q_{\max }$ is precisely $\frac{2}{3}$. The occurrence of the maximum flux at $\theta=0$ expslains why the excess fluid cannot pass through and accumulates below it, and also why the limiting flux is $q=\frac{2}{3}$ (this value can only be changed by higher-order effects, such as surface tension).

Finally, as follows from equation (2.8) for its larger root, $h \approx\left(\theta-\frac{3}{2} \pi\right)^{-1 / 2}$ as $\theta \rightarrow \frac{3}{2} \pi$, which is an integrable singularity. Hence, no matter how close the jump is to the cylinder's bottom, the net mass will not exceed a certain critical value, which can be 

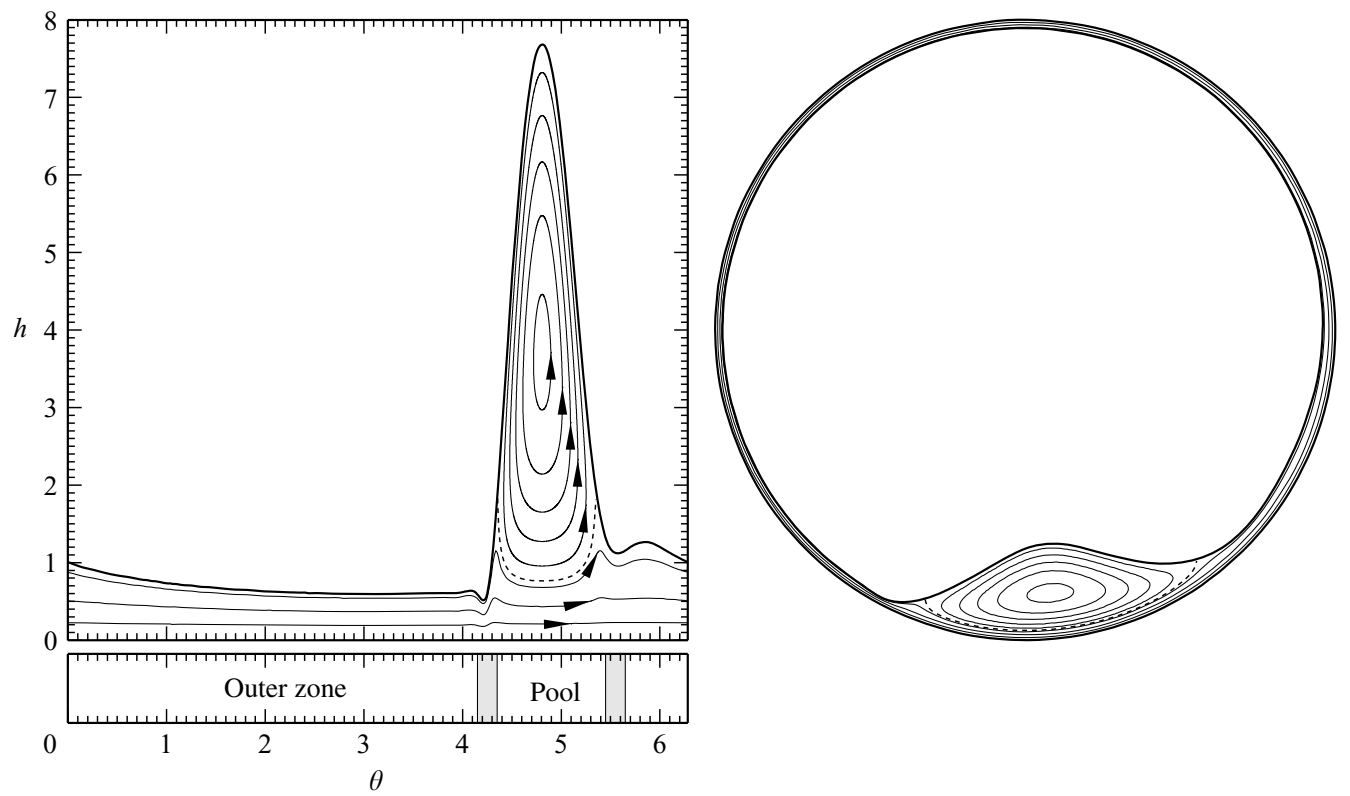

FIGURE 4. Numerical solution of equation (2.5) with $M=9.5, \epsilon=10^{-3}$, and the corresponding streamlines. The dotted line shows the boundary of the re-circulation region. The spatial scale of the right-hand panel corresponds to $\alpha=0.04$, where $\alpha$ is given by (2.2). The lower left panel shows the asymptotic structure of the solution (the transitional regions are shaded).

found through numerical integration of the limiting solution,

$$
M_{* *} \approx 6.93 .
$$

Note that the leading-order equation (2.8) does not have solutions with $M>M_{* *}-$ however, this does not necessarily mean that the full problem (2.5)-(2.7) has no such solutions too. In other words, a steady state with $M>M_{* *}$ may well exist, but it must be crucially affected by surface tension.

\section{Asymptotic analysis of problem (2.5)-(2.7)}

In this section, we shall take advantage of the smallness of $\epsilon$ and examine the problem through matched asymptotics. With this method, it is crucial to correctly guess the solution's asymptotic structure (a task usually assisted by numerical results and physical intuition), then verify the guess by matching the solutions found for different asymptotic zones.

In the present problem, we should expect a pool located about the cylinder's bottom, $\theta \approx \frac{3}{2} \pi$, and strongly affected by surface tension. Because of the cylinder's rotation, fluid is being withdrawn from the pool and taken into the outer zone, $\theta-\frac{3}{2} \pi=O(1)$, where surface tension is weak. The two main zones should be matched in the regions where the film leaves/re-enters the pool (the right/left-hand transitional regions respectively).

In order to make the general picture more comprehensible to the reader, a typical solution (calculated numerically - see $\S 5$ ) and the corresponding streamlines are shown in figure 4 . The asymptotic structure of the solution is shown in the lower left panel. 


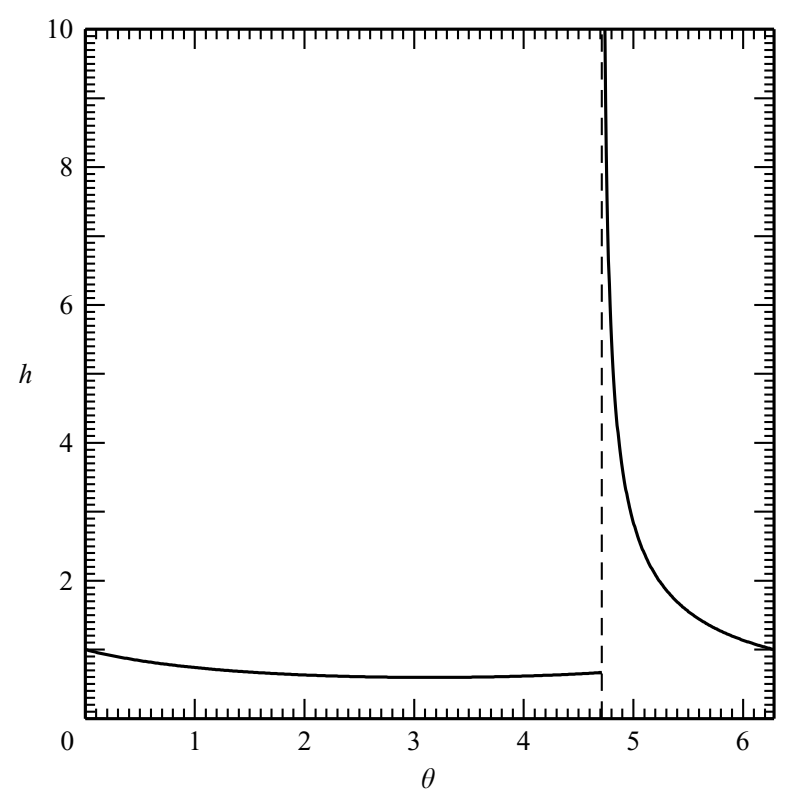

FigURE 5. The outer solution (3.2).

\subsection{The outer zone}

In the outer zone, capillary effects are weak - hence, the full equation (2.5) can be replaced with its truncated version, (2.8). Then, if there is a supply of fluid at the cylinder's bottom (the pool), we can assume that the cylinder's rotation withdraws from it as much fluid as it can, and the flux is close to its limiting value,

$$
q \approx \frac{2}{3}
$$

It is also clear that fluid accumulates on the rising side of the cylinder - hence, the larger root of (2.8), (3.1) should be chosen there.

Thus, in the outer zone,

$$
h \approx \begin{cases}\text { the smaller positive root of }(2.8),(3.1) & \text { if } \theta \in\left[0, \frac{1}{2} \pi\right), \\ \text { the only positive root of }(2.8),(3.1) & \text { if } \theta \in\left[\frac{1}{2} \pi, \frac{3}{2} \pi\right], \\ \text { the larger positive } \operatorname{root} \text { of }(2.8),(3.1) & \text { if } \theta \in\left(\frac{3}{2} \pi, 2 \pi\right] .\end{cases}
$$

Note that, at $\theta=\frac{3}{2} \pi$, the right-hand and left-hand limits of solution (3.2) do not match,

$$
\begin{aligned}
& h \rightarrow \frac{2}{3} \quad \text { as } \quad \theta \rightarrow \frac{3}{2} \pi-0, \\
& h \rightarrow\left(\frac{3}{\theta-\frac{3}{2} \pi}\right)^{1 / 2} \quad \text { as } \quad \theta \rightarrow \frac{3}{2} \pi+0
\end{aligned}
$$

(see figure 5). Thus, in the inner zones, the solution coming from the right will need to be 'brought down' and matched to that coming from the left. 


\subsection{The pool zone}

In the inner zones (i.e. in the 'pool' and transitional regions), it is convenient to replace $\theta$ with

Then, equation (2.5) becomes

$$
x=\theta-\frac{3}{2} \pi
$$

$$
h-\frac{1}{3} h^{3} \sin x+\frac{1}{3} \epsilon h^{3}\left(\frac{\mathrm{d} h}{\mathrm{~d} x}+\frac{\mathrm{d}^{3} h}{\mathrm{~d} x^{3}}\right)=q .
$$

Since all inner zones are located near $x=0$, we can assume, to leading order, $\sin x \approx x$. Note also that the widths of the inner zones are small - hence, the first derivative in (3.5) is much smaller than the third derivative and, thus, can be omitted. Then, replacing the flux with its limiting value (as justified in $\S 3.1$ ), we rewrite (3.5) in the form

$$
h-\frac{1}{3} h^{3} x+\frac{1}{3} \epsilon h^{3} \frac{\mathrm{d}^{3} h}{\mathrm{~d} x^{3}}=\frac{2}{3} .
$$

In what follows, inner zones will be given (positive or negative) numbers, with 0 denoting the pool zone. For example, the stretched variables for the pool are

$$
h_{0}=\frac{h}{H_{0}}, \quad x_{0}=\frac{x}{X_{0}},
$$

where $H_{0}$ and $X_{0}$ are the the pool's characteristic depth and width. We assume that the shape of the pool is determined by the balance of gravity (the second term on the left-hand side of (3.6)) and surface tension (the third term). Then, substituting (3.7) into equation (3.6) and balancing its second and third terms, we obtain

$$
X_{0}=\frac{\epsilon H_{0}}{X_{0}^{3}} .
$$

Physically, the most interesting regime is the one where the pool contains an order-one amount of fluid (i.e. its mass is comparable to that elsewhere in the flow),

$$
H_{0} X_{0}=1 \text {. }
$$

Equations (3.8)-(3.9) yield

$$
H_{0}=\epsilon^{-1 / 5}, \quad X_{0}=\epsilon^{1 / 5} .
$$

Equation (3.10) will be referred to as the supercritical regime as, in this case, the net mass of the flow exceeds $M_{* *}$ by an order-one value. We shall also consider (in $\S 6$ ) a near-critical regime, such that $M \approx M_{* *}$ and $H_{0} X_{0} \ll 1$.

Now, rewrite equation (3.6) in terms of the stretched variables (3.7), (3.10) and omit small terms:

which yields

$$
-x_{0}+\frac{\mathrm{d}^{3} h_{0}}{\mathrm{~d} x_{0}^{3}}=0
$$

$$
h_{0}=\text { const }_{0}+\text { const }_{1} x_{0}+\text { const }_{2} x_{0}^{2}+\frac{1}{24} x_{0}^{4} .
$$

As verified a posteriori, this polynomial can be matched to the solutions in the other zones only if it has two pairs of double roots - in which case (3.12) can be rearranged as

$$
h_{0}=\frac{1}{24}\left(x_{0}^{2}-W^{2}\right)^{2}, \quad-W \leqslant x_{0} \leqslant W,
$$




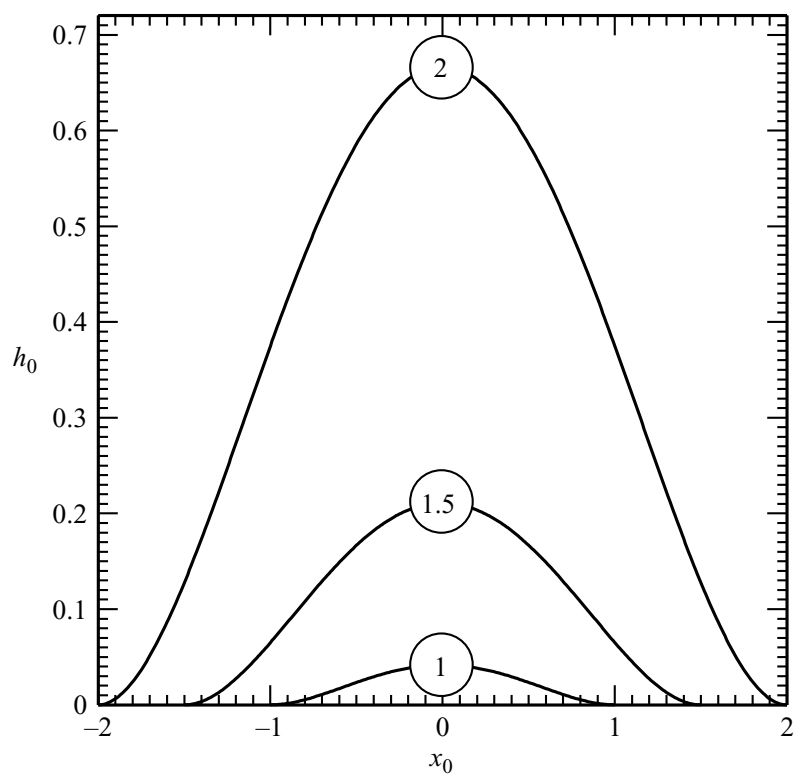

FIGURE 6. The pool solution (3.13) (the curves are marked with the corresponding values of $W$ ).

where $W$ is the scaled half-width of the pool. In the rest of our analysis, $W$ will remain free (i.e. we expect to find a one-parameter family of solutions); $W$ also determines the net mass of the solution,

$$
M \approx M_{* *}+\int_{-\epsilon^{1 / 5} W}^{\epsilon^{1 / 5} W}\left(\epsilon^{-1 / 5} h_{0}\right) \mathrm{d}\left(\epsilon^{1 / 5} x_{0}\right)=M_{* *}+\frac{2}{45} W^{5},
$$

where $M_{* *}$ is the outer-zone's contribution. Examples of solution (3.13) are shown in figure 6.

Finally, we express the asymptotics of (3.13) at the boundaries of the pool in terms of the non-scaled variables,

$$
\begin{array}{ll}
h \approx \frac{1}{6} \epsilon^{-3 / 5} W^{2}\left(x+\epsilon^{1 / 5} W\right)^{2} & \text { for } \quad x \approx-\epsilon^{1 / 5} W, \\
h \approx \frac{1}{6} \epsilon^{-3 / 5} W^{2}\left(x-\epsilon^{1 / 5} W\right)^{2} & \text { for } \quad x \approx \epsilon^{1 / 5} W,
\end{array}
$$

which will be used for matching the pool solution to the neighbouring zones.

\subsection{Discussion}

Observe that the pool solution (3.13) involves a free parameter, $W$, whereas the outer solution (3.2) is entirely fixed, i.e. the latter cannot adjust to various values of the former. The adjustment has to occur in the transitional regions, which should be expected to have fairly complicated structure.

Alternatively, one could try to utilize an outer solution with a non-limiting flux $\left(q<\frac{2}{3}\right)$, so $q$ could adjust to the current value of $W$ (as done by Ashmore et al. (2003), see $\S 7$ ). It turns out, however, that such outer solution cannot be matched to the pool with supercritical parameters (3.10).

Still, since solutions with non-limiting flux might occur in other parameter regimes, they will be examined in $\S 7$. 


\subsection{The left-hand transitional region}

Since Landau \& Levich (1942), the structure of transitional regions between a viscosity-dominated zone and a surface-tension-dominated zone has been examined using the so-called Landau-Levich (LL) equation. In the present problem, it can be obtained from the general equation, (3.6), by neglecting its second (gravity) term,

$$
h+\frac{1}{3} \epsilon h^{3} \frac{\mathrm{d}^{3} h}{\mathrm{~d} x^{3}}=\frac{2}{3} .
$$

Matching the solution of (3.16) to the outer solution (3.3) implies

$$
h \rightarrow \frac{2}{3} \quad \text { as } \quad x \rightarrow-\infty \text {. }
$$

Matching of (3.16) to the pool is less obvious, as none of the three possible asymptotics of the LL equation,

$$
\begin{array}{lll}
h \rightarrow \frac{2}{3} & \text { as } & x \rightarrow+\infty, \\
h \rightarrow \text { const } x^{2} & \text { as } & x \rightarrow+\infty, \\
h \rightarrow x(9 \ln x)^{1 / 3} & \text { as } & x \rightarrow+\infty,
\end{array}
$$

matches the pool solution (3.14). $\dagger$ Still, a zone with the LL equation does exist in the problem at hand, and the correct boundary condition is, in fact, (3.19). There is, however, an auxiliary zone, separating the LL zone from the pool and transforming the linear/logarithmic behaviour of (3.19) into the quadratic behaviour necessary for matching to the pool.

In the remainder of this subsection, the above qualitative picture will be cast into the formal framework of matched asymptotics. The auxiliary and LL zones will be denoted by -1 and -2 respectively.

Anticipating that the logarithm in asymptotics (3.19) will give rise to a logarithmically small parameter, we introduce

$$
\delta=O\left[(\ln \epsilon)^{-1}\right]
$$

Then, the stretched variables for the auxiliary zone are

$$
h_{-1}=\frac{h}{\epsilon^{-1 / 15} \delta^{-2 / 3}}, \quad x_{-1}=\frac{x+\epsilon^{1 / 5} W}{\epsilon^{4 / 15} \delta^{-1 / 3}}
$$

(observe that this zone is 'located' near the pool's left-hand boundary, $x=-\epsilon^{1 / 5} W$ ). Substituting (3.20) into equation (3.6) and omitting small terms, we obtain

$$
\frac{\mathrm{d}^{3} h_{-1}}{\mathrm{~d} x_{-1}^{3}}=0 .
$$

Matching of $h_{-1}$ with the pool requires

$$
h_{-1} \rightarrow \frac{1}{6} W^{2} x_{-1}^{2} \quad \text { as } \quad x_{-1} \rightarrow+\infty,
$$

whereas matching of $h_{-1}$ to the LL zone, as ascertained a posteriori, requires the solution to approach zero with a certain value of its derivative, namely,

$$
\frac{\mathrm{d} h_{-1}}{\mathrm{~d} x_{-1}} \rightarrow 9^{1 / 3} \quad \text { as } \quad h_{-1} \rightarrow 0 .
$$

$\dagger$ Equation (3.18) appears to match the quadratic behaviour of (3.14), but one can verify that the orders of the two solutions do not match. 

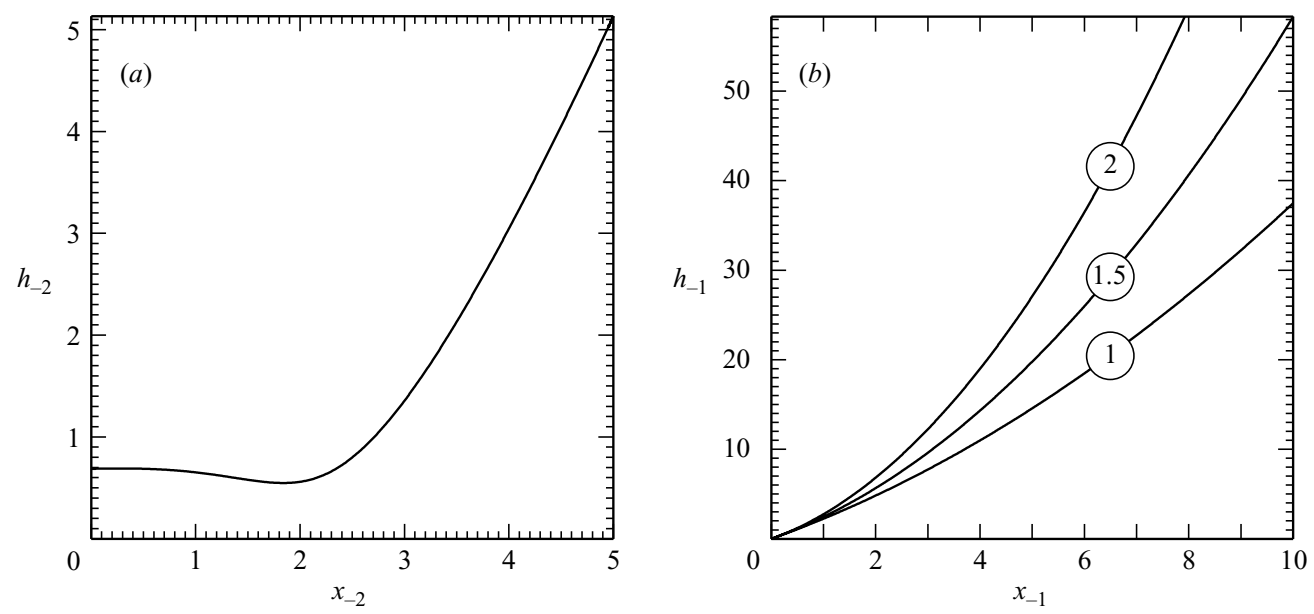

FIGURE 7. The left-hand transitional region: $(a)$ the Landau-Levich zone (3.26)-(3.28);

(b) the auxiliary zone (3.24) (the curves are marked with the corresponding values of $W$ ).

The solution of the boundary-value problem (3.21)-(3.23) is

$$
h_{-1}=9^{1 / 3}\left(x_{-1}+D\right)+\frac{1}{6} W^{2}\left(x_{-1}+D\right)^{2},
$$

where the integration constant $D$ is, essentially, the distance by which zone -1 shifts the left-hand boundary of the pool (it should eventually be related to $W$ ). Examples of solution (3.24) are shown in figure $7 b$.

The stretched variables of the LL zone are

$$
h_{-2}=h, \quad x_{-2}=\frac{x+\epsilon^{1 / 5} W+\epsilon^{4 / 15} \delta^{-1 / 3} D}{\epsilon^{1 / 3}}
$$

(observe that this zone is 'located' near the shifted left-hand boundary of the pool, $x=-\epsilon^{1 / 5} W-\epsilon^{4 / 15} \delta^{-1 / 3} D$ ). As expected, substitution of (3.25) into (3.6) and omission of small terms yields the LL equation,

$$
h_{-2}+\frac{1}{3} h_{-2}^{3} \frac{\mathrm{d}^{3} h_{-2}}{\mathrm{~d} x^{3}}=\frac{2}{3} .
$$

The boundary conditions for $h_{-2}$ are

$$
\begin{array}{lll}
h_{-2} \rightarrow \frac{2}{3} & \text { as } & x_{-2} \rightarrow-\infty, \\
h_{-2} \rightarrow x_{-2}\left(9 \ln x_{-2}\right)^{1 / 3} & \text { as } & x_{-2} \rightarrow+\infty,
\end{array}
$$

which have been discussed above and will be verified below (by matching to the neighbouring zones). The boundary-value problem (3.26)-(3.28) has been solved numerically via 'shooting', and the solution is plotted in figure 7( $a)$.

It remains to ascertain that $h_{-1}$ matches $h_{-2}$. As follows from (3.24),

$$
h_{-1} \rightarrow 9^{1 / 3}\left(x_{-1}+D\right) \quad \text { as } \quad x_{-1} \rightarrow-D,
$$

which, in terms of the non-scaled variables, corresponds to

$$
h \approx 9^{1 / 3} \epsilon^{-1 / 3} \delta^{-1 / 3} \Delta x \quad \text { for } \quad \Delta x=\mathrm{O}\left(\epsilon^{4 / 15} \delta^{-1 / 3}\right),
$$

where

$$
\Delta x=x+\epsilon^{1 / 5} W+\epsilon^{4 / 15} \delta^{-2 / 3} D .
$$


The corresponding expression for zone -2 follows from (3.28) and (3.25),

$$
h \approx \epsilon^{-1 / 3} \Delta x\left[9 \ln \left(\frac{\Delta x}{\epsilon^{1 / 3}}\right)\right]^{1 / 3} \quad \text { for } \quad \Delta x=\mathrm{O}\left(\epsilon^{1 / 3}\right) .
$$

Observe that the logarithmic function in (3.30) corresponds to the logarithmic constant $\delta$ in (3.29).

Since use of van Dyke's principle is not entirely safe for solutions involving logarithms (Hinch 1991, §5.2.6), we shall verify (3.30) via matching by an intermediate variable. Estimating the terms omitted in the derivation of (3.21), one can show that solution (3.24) is applicable for $\Delta x \gg \epsilon^{4 / 15}$ - hence, the intermediate variable can be taken to be

$$
\Delta x=\epsilon^{4 / 15} \delta^{-k} \times \text { const, } \quad k \in\left(-\frac{1}{3}, 0\right) .
$$

Substituting this expression into (3.29) and (3.30) and equating them, one should neglect the logarithms of const and $\delta$, but retain those of $\epsilon$, which yields

$$
\delta=-\frac{15}{\ln \epsilon} .
$$

Thus, (3.29) matches (3.30) subject to $\delta$ satisfying (3.31).

Finally, recall that the 'shift' $D$ of $h_{-1}\left(x_{-1}\right)$ still remains undetermined (see (3.24)); furthermore, the $h_{-2}$ problem (3.26)-(3.28) is invariant with respect to replacing $x_{-2} \rightarrow x_{-2}+$ const. Thus, to fix the 'location' of the solution, we need more boundary conditions, which, however, can only be obtained in the next order, where the equivalents of (3.21) and (3.26) involve the spatial variable. Following Landau \& Levich (1942), and other authors who encountered similar difficulties in similar problems, we shall not carry out this cumbersome calculation, as its result does not affect the leading-order matching. Still, one should keep in mind that the 'true' solutions can be located at an order-one distance from those shown in figure 7 .

\subsection{The right-hand transitional region}

First, observe that, in the vicinity of the pool, the outer solution is strongly asymmetric (see figure 5), which suggests that the right-hand transitional region differs from its left-hand counterpart. This is indeed the case: the latter, for example, includes only two asymptotic zones, whereas the former will be shown to comprise a variable (depending on $\epsilon$ ) number of zones. This is an unusual feature, having both mathematical and physical implications, which will be discussed in this subsection and $\S 4.1$ respectively. Note also that this subsection involves some lengthy calcualtions, so the readers with interests more in physics than mathematics are advised to skip it and move to $\S 4$.

First we shall consider zone 1, located next to the pool. It can be shown that the only set of stretched variables that guarantees the two zones' matching is

$$
h_{1}=\frac{h}{\epsilon^{-1 / 15}}, \quad x_{1}=\frac{x-\epsilon^{1 / 5} W}{\epsilon^{4 / 15}} .
$$

Then, equation (3.6) yields, to leading order,

$$
1+\frac{1}{3} h_{1}^{2} \frac{\mathrm{d}^{3} h_{1}}{\mathrm{~d} x_{1}^{3}}=0
$$

which is similar to the LL equation (3.16). The 'left-hand' boundary condition follows from the matching of (3.32) to the pool asymptotics (3.15),

$$
h_{1} \rightarrow \frac{1}{6} W^{2} x_{1}^{2} \quad \text { as } \quad x_{1} \rightarrow-\infty \text {. }
$$



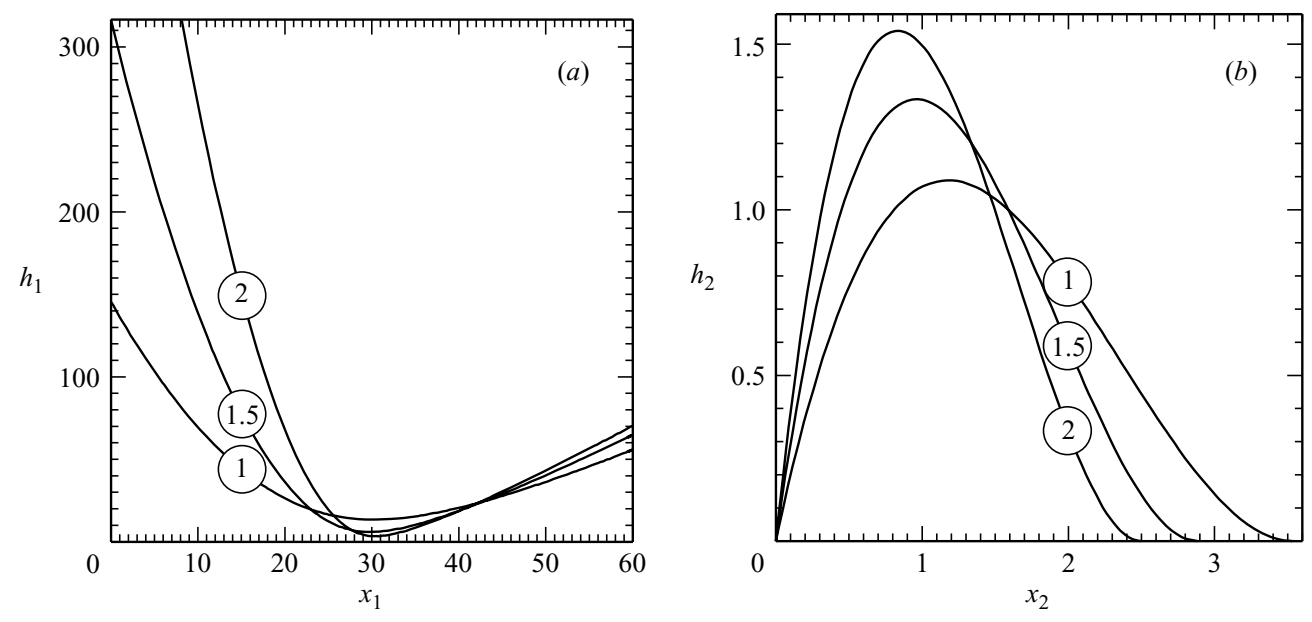

FIGURE 8 . The right-hand transitional region (the curves are marked with the corresponding values of $W)$ : $(a)$ the numerical solution of problem $(3.33)-(3.35) ;(b)$ the explicit solution (3.42)-(3.43).

As $x_{1} \rightarrow+\infty$, equation (3.33) admits either quadratic or linear/logarithmic asymptotics, similar to (3.17) and (3.18) respectively. To choose the correct one, note that, in the next zone, the former gives rise to another peak, with parameters comparable to those of the pool, which has never been observed in numerical simulations (see $\S 6$ ). Thus, we shall assume

$$
h_{1} \rightarrow x_{1}\left(9 \ln x_{1}\right)^{1 / 3} \quad \text { as } \quad x_{1} \rightarrow+\infty .
$$

Thus, $h_{1}$ grows as $x \rightarrow+\infty$, which, again, indicates a peak in the next zone - but the amplitude of this peak will be much smaller than that of the pool, as indeed corroborated by our numerical results.

Problem (3.33)-(3.35) was solved numerically via 'shooting'. Several examples of $h_{1}\left(x_{1}\right)$ are shown in figure $8(a)$.

In the next zone, the stretched variables are

$$
h_{2}=\frac{h}{\epsilon^{-1 / 10} \xi_{2}^{-1 / 2}}, \quad x_{2}=\frac{x-\epsilon^{1 / 5} W}{\epsilon^{7 / 30} \xi_{2}^{-1 / 6}},
$$

where the (logarithmically small) parameter $\xi_{2}$ is related to $\epsilon$ by

$$
\xi_{2}=-\frac{5}{3 \ln \epsilon} \text {. }
$$

Substitution of (3.36) into equation (3.6) yields, to leading order,

$$
-W+\frac{\mathrm{d}^{3} h_{2}}{\mathrm{~d} x_{2}^{3}}=0 .
$$

The solution to this equations is given by a cubic polynomial which, generally, has three roots. However, it can be shown that this zone can be matched to the next one only if the two larger roots coincide. Hence, denoting the resulting double root by $F$, we require

$$
h_{2}=0, \quad \frac{\mathrm{d} h_{2}}{\mathrm{~d} x_{2}}=0 \quad \text { at } \quad x_{2}=F .
$$


Matching of this zone with the previous one is similar to that of zones -1 and -2 , and yields

$$
h_{2}=0, \quad \frac{\mathrm{d} h_{2}}{\mathrm{~d} x_{2}}=9^{1 / 3} \quad \text { at } \quad x_{2}=G,
$$

where $G$ is the solution's smaller root. Finally, observe that the previous zone, zone 1, is much narrower than either of its neighbours, zone 2 and the pool - compare (3.32) to (3.36) and (3.7), (3.10). Thus, to leading order, we shall require that the left-hand boundary of zone 2 coincide with the right-hand boundary of the pool, i.e.

$$
G=0 .
$$

The solution of (3.38)-(3.41) is

$$
h_{2}=\frac{1}{6} W x_{2}\left(x_{2}-F\right)^{2}, \quad 0 \leqslant x_{2} \leqslant F,
$$

where

$$
F=\frac{2^{1 / 2} 3^{5 / 6}}{W^{1 / 2}}
$$

is the width of this zone. Examples of this solution are shown in figure $8(b)$. Observe also that (3.42)-(3.43) imply

$$
h_{2} \rightarrow \frac{W^{1 / 2}}{2^{1 / 2} 3^{1 / 6}}\left(x_{2}-F\right)^{2} \quad \text { as } \quad x_{2} \rightarrow F,
$$

which we shall use when matching this zone to the next one.

The next zone's scaling is, in fact, fully determined by matching to (3.44),

$$
h_{3}=\frac{h}{\epsilon^{-1 / 10} \xi_{2}^{1 / 6}}, \quad x_{3}=\frac{x-\epsilon^{1 / 5} W_{3}}{\epsilon^{7 / 30} \xi_{2}^{1 / 6}},
$$

where

$$
W_{3}=W-\epsilon^{1 / 30} \xi_{2}^{-1 / 6} F
$$

(the second term in $W_{3}$ reflects the fact that zones 1 and 3 are 'separated' by the width of zone 2). Then, the boundary-value problem for $h_{3}$ is

$$
\begin{array}{rlrl}
1+\frac{1}{3} h_{3}^{2} \frac{\mathrm{d}^{3} h_{3}}{\mathrm{~d} x_{3}^{3}}=0, & \\
h_{3} \rightarrow \frac{W^{1 / 2}}{2^{1 / 2} 3^{1 / 6}} x_{3}^{2} & \text { as } & x_{1} \rightarrow-\infty, \\
h_{3} \rightarrow x_{3}\left(9 \ln x_{3}\right)^{1 / 3} & \text { as } & x_{1} \rightarrow+\infty .
\end{array}
$$

Comparison of these equations with (3.33)-(3.35) shows that $h_{3}$ can be obtained by re-scaling $h_{1}$.

Zones 2 and 3 are followed by a sequence of similar asymptotic zones: the evennumbered ones are described by the explicit solution (3.42)-(3.43); and the oddnumbered ones, by the boundary-value problem (3.46 )-(3.48). The corresponding stretched variables are

$$
\begin{aligned}
h_{2 n} & =\frac{h}{\epsilon^{-1 / 10} \xi_{2 n}^{-1 / 2}}, & x_{2 n} & =\frac{x-\epsilon^{1 / 5} W_{2 n-2}}{\epsilon^{7 / 30} \xi_{2 n}^{-1 / 6}} & \text { for } & 2 n \geqslant 4, \\
h_{2 n+1} & =\frac{h}{\epsilon^{-1 / 10} \xi_{2 n}^{1 / 6}}, & x_{2 n+1} & =\frac{x-\epsilon^{1 / 5} W_{2 n}}{\epsilon^{7 / 30} \xi_{2 n}^{1 / 6}} & \text { for } & 2 n+1 \geqslant 3,
\end{aligned}
$$


where

$$
W_{2 n}=W-\epsilon^{1 / 30}\left(\xi_{2}^{-1 / 6}+\xi_{4}^{-1 / 6}+\cdots+\xi_{2 n}^{-1 / 6}\right) F
$$

is the cumulative width of the even-numbered zones (the odd ones are much narrower). $\xi_{2 n}$ are linked recursively to $\xi_{2 n-2}$ (and, eventually, to $\xi_{2}$ ) by

$$
\xi_{2 n}=-\frac{6}{\ln \xi_{2 n-2}} \text {. }
$$

To visualize the asymptotic structure of the solution, observe that, as follows from (3.49), the characteristic heights and widths of the peaks located in even-numbered zones are

$$
H_{2 n}=\epsilon^{-1 / 10} \xi_{2 n}^{-1 / 2}, \quad X_{2 n}=\epsilon^{7 / 30} \xi_{2 n}^{-1 / 6} .
$$

The corresponding parameters of the troughs (located in odd-numbered zones) can be derived from (3.50),

$$
H_{2 n+1}=\epsilon^{-1 / 10} \xi_{2 n}^{1 / 6}, \quad X_{2 n+1}=\epsilon^{7 / 30} \xi_{2 n}^{1 / 6} .
$$

Note also that equations (3.37) and (3.51) imply that

$$
\xi_{2}=O\left(\frac{1}{\ln \epsilon}\right), \quad \xi_{4}=O\left(\frac{1}{\ln \ln \epsilon}\right), \quad \xi_{6}=O\left(\frac{1}{\ln \ln \ln \epsilon}\right), \quad \ldots,
$$

i.e. $\xi_{2 n}$ increase with $n$ and, hence, the peaks become smaller and narrower, while the troughs become shallower and wider. Sooner or later (say, at $n=N$ ), $\xi_{2 N}$ becomes comparable to unity, and the corresponding peak and trough are indistinguishable, as

$$
H_{2 N+1}=H_{2 N}=\epsilon^{-1 / 10}, \quad X_{2 N+1}=X_{2 N}=\epsilon^{7 / 30} .
$$

Thus, we need to introduce a 'limiting' asymptotic zone, with the stretched variables given by

$$
h_{2 N}=\frac{h}{\epsilon^{-1 / 10}}, \quad x_{2 N}=\frac{x-\epsilon^{1 / 5} W_{2 N}}{\epsilon^{7 / 30}} .
$$

Substituting (3.52) into (3.6) and omitting small terms, we obtain

$$
h_{2 N}-\frac{1}{3} W h_{2 N}^{3}+\frac{1}{3} h_{2 N}^{3} \frac{\mathrm{d}^{3} h_{2 N}}{\mathrm{~d} x_{2 N}^{3}}=0 .
$$

The matching of $h_{2 N}$ to the outer solution (3.4) requires

$$
h_{2 N} \rightarrow\left(\frac{3}{W}\right)^{1 / 2} \quad \text { as } \quad x_{2 N} \rightarrow+\infty .
$$

Before we discuss the left-hand boundary condition, observe that the previous equations (3.33) and (3.38) are both included in (3.53) as limiting cases, as they can be derived from it by re-scaling the variables. In fact, the solutions of the previous zones are simply an asymptotic description of $h_{2 N}$ for large negative values of $x_{2 N}$. Thus, we should require that, as $x_{2 N} \rightarrow-\infty, h_{2 N}$ 'consists' of a sequence of maxima and minima described by (3.42)-(3.43) and (3.46)-(3.48).

In practice, however, we just need to integrate equation (3.53) from left to right, starting from a maximum, and show that, eventually (at the positive infinity), we can satisfy condition (3.54). Furthermore, since the spatial variable $x_{2 N}$ does not explicitly appear in (3.53)-(3.54), we can start integration from $x_{2 N}=0$, i.e. the left-hand 


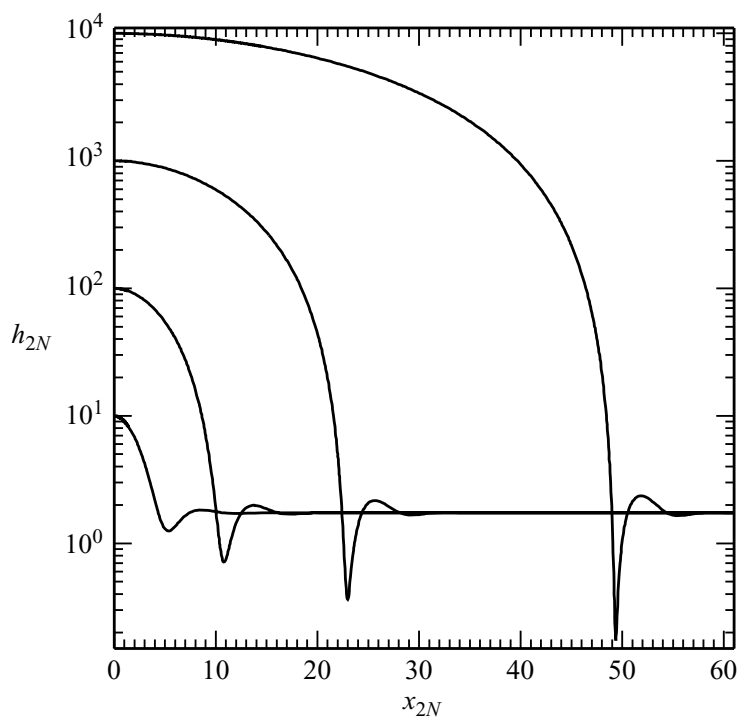

FIGURE 9. The numerical solution of the boundary-value problem (3.53)-(3.55) for $W=1$, $A=10,100,1000,10000$.

boundary condition amounts to

$$
h_{2 N}=A, \quad \frac{\mathrm{d} h_{2 N}}{\mathrm{~d} x_{2 N}}=0 \quad \text { at } \quad x_{2 N}=0,
$$

where $A$ is a large positive constant.

It turned out that the boundary-value problem (3.53)-(3.55) cannot be solved numerically through 'shooting', owing to the exponential dependence of the solution on the initial condition and other complicating factors. As a result, we used the same iterative procedure as used for the exact boundary-value problem (2.5)-(2.6) (see $\S 5.1)$. The results are shown in figure 9: one can see that, no matter how large the amplitude of the initial maximum is, the solution invariably reaches the correct limiting value as $x_{2 n} \rightarrow+\infty$. It also has the correct (oscillating) structure, with a narrow minimum following a wide maximum.

Effectively, the boundary-value problem (3.53)-(3.55) describes all of the right-hand transitional region starting from zone 1.

\section{Discussion}

4.1. The right-hand transitional region from a physical viewpoint

Mathematically, the most interesting aspect of the above solution is the variable number of asymptotic zones in the right-hand transitional region (recall that $N$ depends on how small $\epsilon$ is). This unusual feature has been found earlier for a similar setting - namely, a liquid film sliding down a vertical wall into a pool (Wilson \& Jones 1983). In that case, the asymptotic structure of the solution also involved indefinitely many zones, but, in the exact solution, only one or two peaks (capillary ripples) could be observed. The discrepancy was due to the fact that the sequence of formally small parameters which determined the zones rapidly increased, so in some cases even the second one was comparable to unity. 


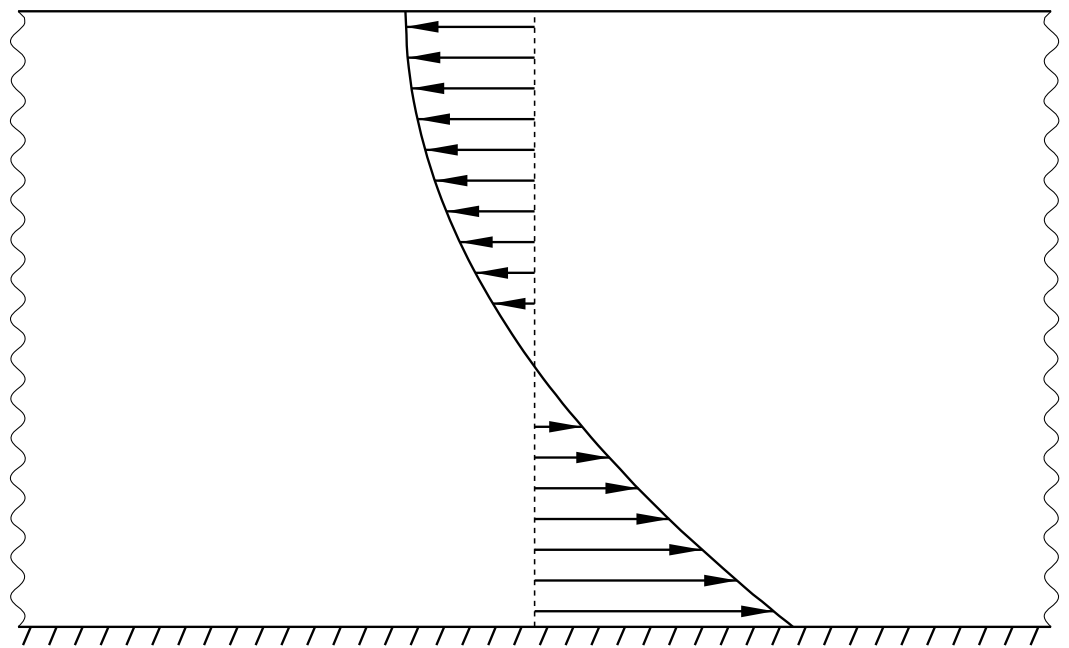

FIGURE 10. The azimuthal velocity profile (4.2) of a rimming flow.

In the present case, the sequence of small parameters $\xi_{2 n}$, on which the separation of scales of the asymptotic zones is based, increase exponentially, i.e. even faster than those in the work of Wilson \& Jones (1983). For $\epsilon=10^{-12}$, for example, equation (3.37) yields $\xi_{2} \approx 0.0603$ which is indeed small, but then (3.51) with $n=2$ yields $\xi_{4} \approx 2.13=O(1)$. Thus, few ripples should be expected in the numerical solution of the present problem (see the next section).

Note also that Wilson \& Jones (1983) observed ripples on a film entering a pool, whereas a film being withdrawn from a pool turned out to be smooth (Wilson 1982). This is at odds with the present case, where the variable number of zones (physically, corresponding to ripples) were observed in the right-hand transitional region, where the film is being withdrawn from the pool.

To resolve the contradiction, we need the expression for the non-dimensional azimuthal velocity $v$, i.e. the component tangential to the cylinder's wall, as a function of the film's non-dimensional depth $z$ (see Appendix A, formula (A 2)). Then, since $h$ satisfies the steady-state equation (2.8), expression (A 2) can be rearranged into

$$
v=1+\left(\frac{z^{2}}{2}-z h\right) \frac{3(h-q)}{h^{3}} .
$$

Now, recall that, in both transitional regions, $h \gg, q$, and hence (4.1) becomes

$$
v \approx 1+\left(\frac{z^{2}}{2}-z h\right) \frac{3}{h^{2}} .
$$

The dependence $v$ vs. $z$ is shown in figure 10. One can see that, even though the net flux $q$ is positive (directed to the right), the near-surface velocity is directed to the left.

Given that capillary ripples are most sensitive to what happens near the fluid's surface, it comes as no surprise that they occur in the right-hand transitional region, where the near-surface layer flows towards the pool - just as it does in the setting considered by Wilson \& Jones (1983). 


\subsection{The applicability of the solution obtained}

The lubrication theory, on which all results in this paper are based, requires the film to be thin and its slope small. Thus, we should make sure that our asymptotic solution complies with these requirements.

First, estimating the depth of the pool (where the film is thickest) from (3.10) and also recalling how $h$ was non-dimensionalized (see (2.1)), we obtain

$$
\alpha \epsilon^{-1 / 5} \ll 1
$$

Secondly, it can be verified that the steepest slope occurs in zone -1 of the left-hand transitional region. Extracting the scales for $h$ and $x$ from (3.20) and (3.31),

$$
H=\epsilon^{-1 / 15}\left(-\frac{15}{\ln \epsilon}\right)^{-2 / 3}, \quad X=\epsilon^{4 / 15}\left(-\frac{15}{\ln \epsilon}\right)^{-1 / 3}
$$

and estimating the slope as $\alpha H / X$, we obtain

$$
\alpha \epsilon^{-1 / 3}\left(-\frac{15}{\ln \epsilon}\right)^{-1 / 3} \ll 1
$$

Note also that, apart from surface tension, rimming flows are affected by hydrostatic pressure. The latter can be described using the lubrication theory (Benjamin et al. 1993), resulting in

$$
h-\frac{1}{3} h^{3} \cos \theta+\frac{1}{3} \epsilon h^{3}\left(\frac{\mathrm{d} h}{\mathrm{~d} \theta}+\frac{\mathrm{d}^{3} h}{\mathrm{~d} \theta^{3}}\right)+\alpha\left(\frac{1}{3} h^{3} \frac{\mathrm{d} h}{\mathrm{~d} \theta} \sin \theta+\frac{1}{2} h^{4} \cos \theta-\frac{1}{2} h^{2}\right)=q,
$$

where $\alpha$ is given by (2.2). By comparison with (4.5), our equation (2.5) misses the expression involving $\alpha$; hence, to validate our results, we need that to be much smaller than the largest of 'our' terms. In the outer zone, where the solution is determined by the first two terms of (4.5), this implies $\alpha \ll 1$, which condition is weaker than (4.3)-(4.4) and, thus, can be discarded. In the inner zones, in turn, pressure should be compared to surface tension (which is always a leading-order effect); one should also keep in mind that the spatial scale $X$ of the solution is small. Comparing the largest of the surface-tension terms (the second one) to the largest of the pressure terms (the first one), we obtain

$$
\frac{\epsilon}{X^{2}} \gg \alpha
$$

Condition (4.6) is most stringent in the inner zone with the largest $X$, which is zone $2 N$ of the right-hand transitional region. Substituting $X=X_{2 N}=\epsilon^{7 / 30}$ into (4.6), we obtain

$$
\epsilon^{8 / 15} \gg \alpha
$$

This condition is stronger than (4.3)-(4.4) and, thus, can be regarded as the sole applicability criterion of our results. Note, however, that all of the above conditions are 'local': if they do not hold, this affects only some of the asymptotic zones, while the solution in other zones remains intact. 


\section{Numerical results}

\subsection{The method}

The simplest way to compute the solution of the steady-state equation (2.5) is to simulate the evolutionary equation (2.4) with a suitable initial condition, say,

$$
h=\text { const } \quad \text { at } \quad t=0 .
$$

In the limit $t \rightarrow \infty$, the solution becomes steady and, by varying the constant in (5.1), one can compute steady states for various values of the net mass $M$. This approach was used by Ashmore et al. (2003), and we also attempted to use a somewhat enhanced version of it (with the implicit Euler method for the time derivative, fivepoint second-order upwind discretization of spatial derivatives, and a sophisticated mesh refinement algorithm).

However, even though simulating the evolution equation (2.4) yields an accurate solution for $h$, it does not for the flux $q$. The problem is that, to calculate $q$, one needs to compute $\partial^{3} h / \partial \theta^{3}$, the accuracy of which is much lower than that of $h$. Moreover, since all interesting particular cases in this problem are concentrated near $q \approx \frac{2}{3}$, a high-accuracy computation of this quantity is essential.

As a result, we solved the steady-state problem (2.5)-(2.7) for $h$ and $q$ (for a given mass $M$ ) via an iterative procedure based on Newton linearization. Equation (2.5) was divided by $\frac{1}{3} h^{3}$, then replaced with a linear ODE,

$$
\begin{aligned}
\frac{3}{h_{n}^{2}}-\frac{6\left(h_{n+1}-h_{n}\right)}{h_{n}^{3}}-\cos \theta+\epsilon\left(\frac{\mathrm{d} h_{n+1}}{\mathrm{~d} \theta}+\frac{\mathrm{d}^{3} h_{n+1}}{\mathrm{~d} \theta^{3}}\right) \\
=\frac{3 q_{n}}{h_{n}^{3}}-\frac{9 q_{n}\left(h_{n+1}-h_{n}\right)}{h_{n}^{4}}+\frac{3\left(q_{n+1}-q_{n}\right)}{h_{n}^{3}}
\end{aligned}
$$

where $\left(h_{n}, q_{n}\right)$ represent the 'current' iteration and $\left(h_{n+1}, q_{n+1}\right)$, the next one. Equation (5.2) was discretized using the five-point (fourth-order) symmetric approximations of the derivatives and solved together with the discrete equivalents of

$$
\begin{gathered}
h_{n+1}(\theta+2 \pi)=h_{n+1}(\theta), \\
\int_{0}^{2 \pi} h_{n+1}(\theta) \mathrm{d} \theta=M
\end{gathered}
$$

through Crout's UL-decomposition algorithm (see Press et al. 1992). This approach turned out to be faster and more accurate than simulating the evolution equation (2.4).

In some cases, where a small change in $M$ gives rise to a large change in $q$, it is more convenient to solve equation (2.5) for a given $q$, without imposing condition (2.7). In this case, the last term in equation (5.2) should be omitted and condition (5.4) discarded.

\subsection{The results}

A typical pool solution and the corresponding streamlines are shown in figure 4. One can see that most of the pool is occupied by a large re-circulation area.

Using the computed solution, we have also verified the accuracy of the asymptotic outer solution (3.2); it turned out that, for $\epsilon=10^{-4}-10^{-3}$, the asymptotic and numerical solutions are indistinguishable everywhere except for the pool zone (see figure $11 a$ )

The agreement between the numerical and asymptotic solutions for the pool (see figure $11 b$ ) is worse than that for the outer solution. To understand why this is so, 

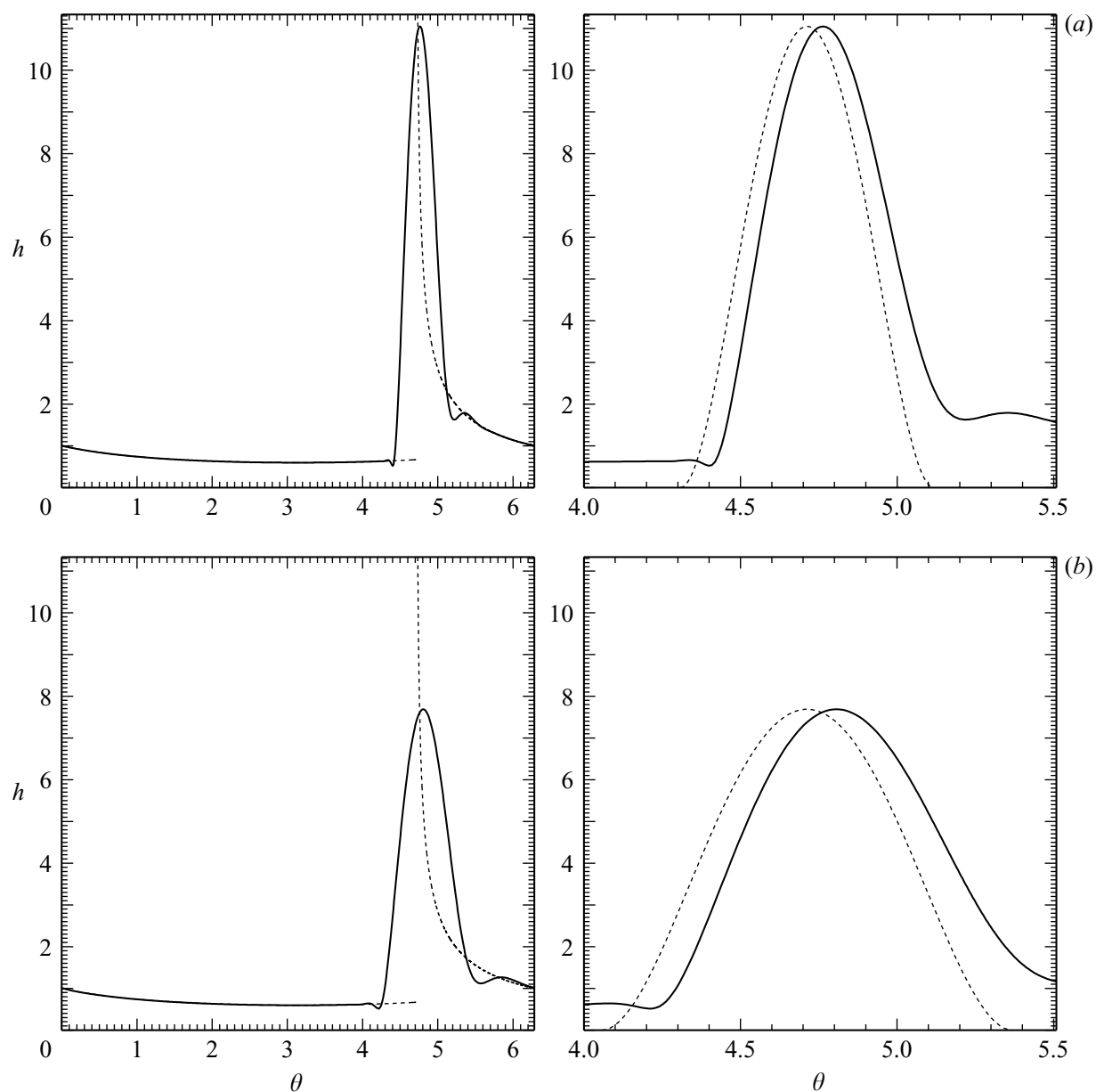

FIGURE 11. Numerical solution of the exact equation (2.4) with $M=9.5$ and $(a) \epsilon=10^{-4},(b)$ $\epsilon=10^{-3}$. The dotted line shows the outer asymptotic solution (3.2) (the left-hand panels) and the pool solution (3.13) (the right-hand panels).

note that the accuracy of the former is $\epsilon^{1 / 5}$ (which can be verified by substituting the stretched variables (3.7), (3.10) into equation (3.6) and estimating the magnitude of the next-to-leading-order term). The accuracy of the outer solution, in turn, is $\epsilon$, which is much higher.

However, the relatively low accuracy of the asymptotic solution for the pool manifests itself, mainly, in the pool's incorrect position (see figure 11b), whereas its shape is predicted quite well. To illustrate this, we introduce the width $w_{0.5}$ of the pool at half of the maximum depth $h_{\max }$, and similar widths, $w_{0.7}$ and $w_{0.9}$, at $0.7 h_{\max }$ and $0.9 h_{\max }$ (see figure $12 a$ ). These parameters are plotted as functions of $h_{\max }$ alongside their asymptotic counterparts (based on solution (3.13)) in figure 12(b). Clearly, the numerical and asymptotic results agree reasonably well even for $\epsilon=10^{-3}$ (note that the neighbouring regions affect $w_{0.5}$ more than the other two widths; hence, the accuracy of its asymptotic value is the lowest). The disagreement between the numerical and asymptotic positions of the pool will be qualitatively explained in the next section. Note also that no more than one ripple has been observed in the 

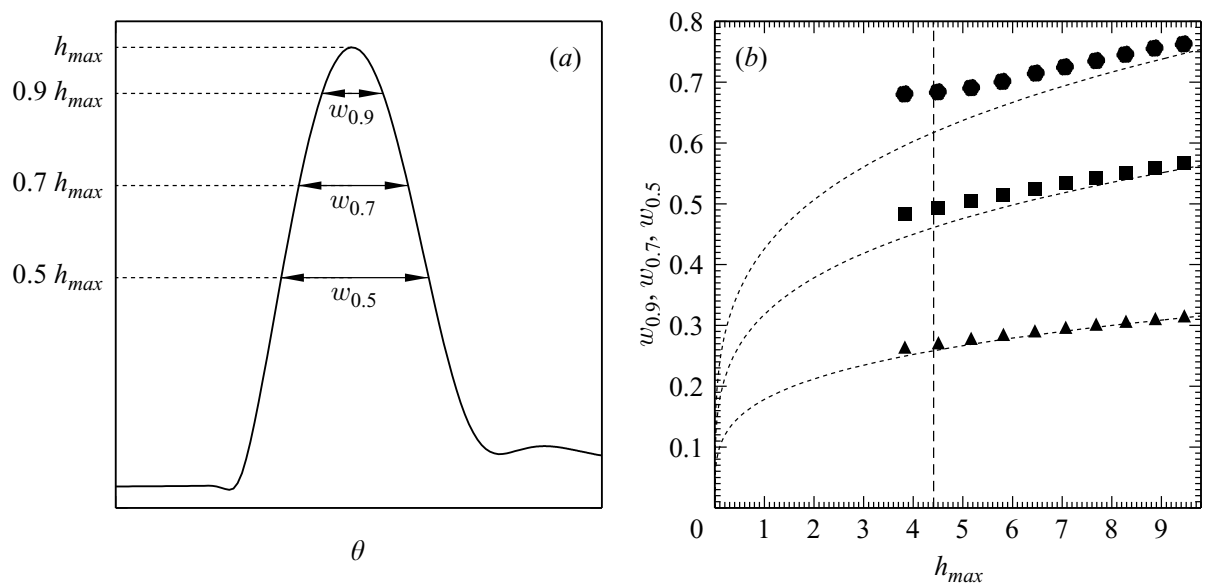

Figure 12. The 'shape' of the pool. (a) The definitions of $w_{0.9}, w_{0.7}$, and $w_{0.5} ;(b) w_{0.9}, w_{0.7}$, and $w_{0.5}$ (the triangles, squares, and circles respectively) vs. $h_{\max }$, for the numerical solution of the exact equation (2.5) for $\epsilon=10^{-3}$. The dotted lines show the corresponding asymptotic results based on the pool solution (3.13). The vertical dashed line separates solutions with a shock from those with a pool.

right-hand transitional region, and sometimes (for larger $\epsilon$ and smaller $M$ ) we did not observe any ripples at all.

\section{The near-critical regime}

Note that the maxima of subcritical shock solutions (for which $M<M_{* *}$ ) are positioned inside the fourth quadrant - see figure $3(a)$. On the other hand, the maxima of the supercritical solutions constructed in $\S 3$ (for which $M>M_{* *}$ ) are located at exactly $\theta=\frac{3}{2} \pi$. Thus, to trace how the former solutions transform into the latter, one should consider a near-critical regime, for which $M \approx M_{* * *}$. It should be the most general regime, such that the 'neighbouring' ones can be obtained as its limiting cases.

It can be verified a posteriori that the most general regime corresponds to the following scaling of the pool:

$$
h_{0}=\frac{h}{\epsilon^{-1 / 9} \gamma^{-4 / 9}}, \quad x_{0}=\frac{x}{\epsilon^{2 / 9} \gamma^{-1 / 9}},
$$

where the (logarithmically) small parameter $\gamma$ is

$$
\gamma=-\frac{9}{\ln \epsilon} \text {. }
$$

Substitution of (6.1) into (3.6) yields the same equation and solution as in the supercritical regime, i.e. (3.11) and (3.12) respectively. Even the right-hand boundary condition is the same, i.e. we should require that the solution approach zero with zero derivative. At the left-hand boundary, however, the derivative should be equal to $9^{1 / 3}$ (which can be shown to be the only choice that ensures matching to the left-hand transitional region). 


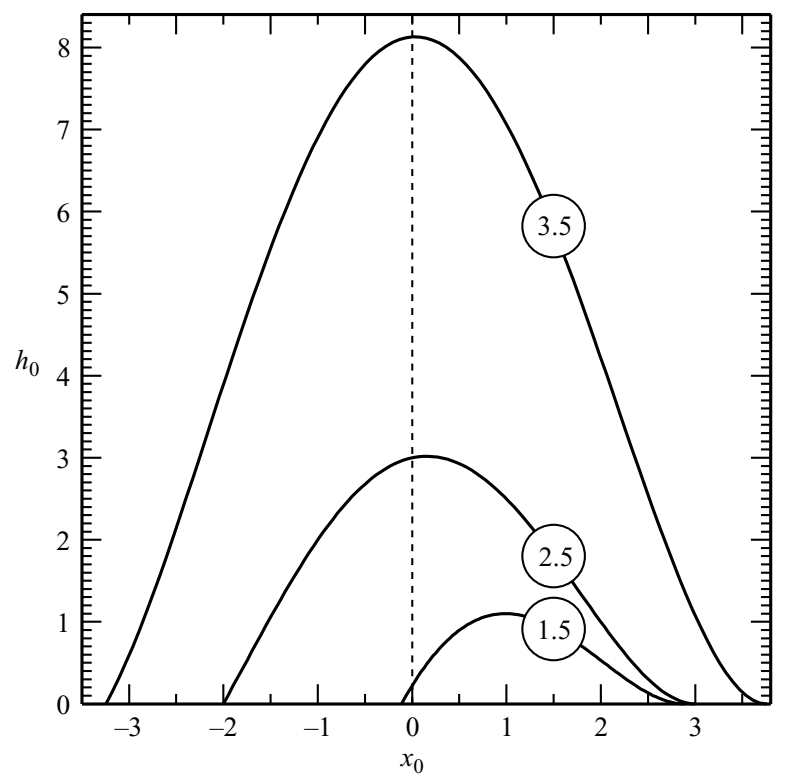

FIGURE 13. The pool solution (6.5), (6.7) for the near-critical regime (the curves are marked with the corresponding values of $W^{\prime}$ ).

Thus, denoting the left-hand and right-hand boundaries of the pool by $A$ and $B$, we require

$$
\begin{array}{llll}
\frac{\mathrm{d} h_{0}}{\mathrm{~d} x_{0}}=9^{1 / 3}, & h_{0}=0 & \text { at } & x=A, \\
\frac{\mathrm{d} h_{0}}{\mathrm{~d} x_{0}}=0, & h_{0}=0 & \text { at } & x=B .
\end{array}
$$

Under conditions (6.3)-(6.4), solution (3.12) becomes

$$
h_{0}=\frac{1}{24}\left(x_{0}-A\right)\left(x_{0}+A+2 B\right)\left(x_{0}-B\right)^{2}, \quad A \leqslant x_{0} \leqslant B,
$$

where $A$ and $B$ satisfy

$$
\frac{1}{12}(A+B)(A-B)^{2}=9^{1 / 3} .
$$

It is convenient to introduce here $W^{\prime}=\frac{1}{2}(B-A)$, where $W^{\prime}$ is the scaled half-width of the pool (the equivalent of $W$ introduced for supercritical solutions). Then, (6.6) can be satisfied by putting

$$
A=\frac{3^{5 / 3}}{2 W^{\prime 2}}-W^{\prime}, \quad B=\frac{3^{5 / 3}}{2 W^{\prime 2}}+W^{\prime} .
$$

As $W^{\prime} \rightarrow 0$, solution (6.5), (6.7) moves to the right, while its amplitude decays (see figure 13) - this limit describes solutions with shocks located near the bottom of the cylinder. In the opposite limit, $W^{\prime} \rightarrow \infty$, solution (6.5), (6.7) becomes more symmetric and tends to the supercritical pool solution (3.13).

The structure of the transitional regions for the near-critical regime is similar to that of the supercritical one with one exception: the left-hand transitional region of the former consists of a single zone. This zone is described by the Landau-Levich equation, the linear/logarithmic solution (3.28) of which matches the pool exactly, so there is no need for an auxiliary zone. 
It should be noted, however, that the near-critical solution has a much lower accuracy than the supercritical one, as the parameter $\gamma$ on which the former is based is rarely small (if, for example, $\epsilon=10^{-6}$, then (6.2) yields $\gamma \approx 0.65$, which can hardly be regarded as small). Thus, solution (6.5), (6.7) should, rather, be viewed as a qualitative aid for explaining subtle features of rimming flows, such as the above-mentioned shift of the pool zone, etc.

\section{Comparison with Ashmore et al. (2003)}

Before discussing the paper by Ashmore et al. (2003, henceforth referred to as AHS), we shall rewrite the pool solution (3.13) in terms of the original non-scaled variables (see (3.7)-(3.8)) and the maximum depth $h_{\max }=\frac{1}{24} H_{0} W^{4}$ of the pool,

$$
h=h_{\max }\left[\frac{x^{2}}{\left(24 \epsilon h_{\max }\right)^{1 / 2}}-1\right]^{2}, \quad-\left(24 \epsilon h_{\max }\right)^{1 / 4} \leqslant x \leqslant\left(24 \epsilon h_{\text {max }}\right)^{1 / 4} .
$$

Then, at the boundaries of the pool, we have

$$
\begin{aligned}
& h \rightarrow\left(\frac{2 h_{\max }}{3 \epsilon}\right)^{1 / 2}\left[x+\left(24 \epsilon h_{\max }\right)^{1 / 4}\right]^{2} \quad \text { as } \quad x \rightarrow-\left(24 \epsilon h_{\max }\right)^{1 / 4}, \\
& h \rightarrow\left(\frac{2 h_{\max }}{3 \epsilon}\right)^{1 / 2}\left[x-\left(24 \epsilon h_{\max }\right)^{1 / 4}\right]^{2} \quad \text { as } \quad x \rightarrow\left(24 \epsilon h_{\max }\right)^{1 / 4},
\end{aligned}
$$

which are equivalent to (3.14)-(3.15) (with $h_{\max }$ replacing $W$ as the free parameter of our family of solutions).

The pool solution (7.1) agrees with the corresponding solution of AHS, but their outer solution does not agree with our (3.2). This is a result of AHS's assumption that the flux is small,

$$
q \ll 1 \text {, }
$$

as opposed to our hypothesis $q \approx \frac{2}{3}$ (see $\S 3.3$ ). Under assumption (7.4), the solution of equation (2.5) can be sought in the form of a series in powers of $q$,

$$
h=q+\mathrm{O}\left(q^{3}\right),
$$

i.e. the film's thickness in the outer zone is almost uniform.

AHS then, effectively, used the assumption

$$
q=\epsilon^{-1 / 6} h_{\max }^{-1 / 2} \hat{q},
$$

where $\hat{q}$ is an order-one constant to be determined later. Keeping (7.6) in mind, consider the right-hand transitional region (unlike our case, it includes only one zone). It can be verified a posteriori that the stretched variables there are

$$
h_{1}=\frac{h}{\epsilon^{-1 / 6} h_{\max }^{-1 / 2}}, \quad x_{1}=\frac{x-\left(24 \epsilon h_{\max }\right)^{1 / 4}}{\epsilon^{1 / 6} h_{\max }^{-1 / 2}} .
$$

The matching of (7.7) to the outer and pool solutions, (7.5)- (7.6) and (7.3), requires

$$
\begin{array}{lll}
h_{1} \rightarrow \hat{q} & \text { as } & x_{1} \rightarrow+\infty, \\
h_{1} \rightarrow\left(\frac{2}{3}\right)^{1 / 2} x_{1}^{2} & \text { as } & x_{1} \rightarrow-\infty .
\end{array}
$$

Next, substituting (7.7) into (3.6), we obtain

$$
h_{1}+\frac{1}{3} h_{1}^{3} \frac{\mathrm{d}^{3} h_{1}}{\mathrm{~d} x_{1}^{3}}=\hat{q} .
$$




\begin{tabular}{|c|c|c|c|c|}
\hline & Type of solution & $M$ & $h_{\max }$ & $q$ \\
\hline (a) & continuous & $M \leqslant M_{*}$ & $h_{\max }=O(1)$ & $q \leqslant \frac{2}{3}$ \\
\hline (b) & shock & $M \in\left(M_{*}, M_{* *}\right)$ & $h_{\max }=O(1)$ & $q=\frac{2}{3}$ \\
\hline (c) & near-critical & $M \approx M_{* *}$ & $h_{\max }=O\left(\epsilon^{-1 / 9} \gamma^{-4 / 9}\right)$ & $q \approx \frac{2}{3}$ \\
\hline (d) & supercritical & $M=M_{* *}+O(1)$ & $h_{\max }=O\left(\epsilon^{-1 / 5}\right)$ & $q \approx \frac{2}{3}$ \\
\hline (e) & strongly supercritical & $M \gg \epsilon^{-1 / 6}$ & $h_{\max } \gg O\left(\epsilon^{-1 / 3}\right)$ & $q \ll 1$ \\
\hline
\end{tabular}

It can be shown that the boundary-value problem (7.8)-(7.10) has a solution only for a certain value of $\hat{q}$, which can be found numerically, $\hat{q} \approx 0.82$ (see Appendix B). Then, (7.6) yields the following expression for the flux:

$$
q \approx 0.82 \epsilon^{-1 / 6} h_{\max }^{-1 / 2} .
$$

This result is compared to the numerical solution of the exact problem in figure 14(b), below.

Observe also that (7.11) is consistent with the small- $q$ assumption (7.4) only if

$$
h_{\max } \gg \epsilon^{-1 / 3} \text {. }
$$

In terms of the net mass (which is mainly determined by the pool solution (7.1)), this restriction has the form

$$
M \gg \epsilon^{-1 / 6} .
$$

Naturally, since we assumed $q=\frac{2}{3}$, our results are applicable in the opposite limit,

$$
h_{\max } \ll \epsilon^{-1 / 3}, \quad M \ll \epsilon^{-1 / 6} .
$$

One can see that the present work is complementary to AHS's analysis.

\section{Concluding remarks: the complete classification of rimming flows with surface tension}

Steady rimming flows with surface tension are governed by equation (2.5) and are characterized by two parameters: the non-dimensional capillary coefficient $\epsilon$ defined by (2.3) and the non-dimensional mass $M$ defined by (2.7).

A classification of rimming flows depending on $M$ and $\epsilon$ is presented in table 1 and briefly summarized below (the numbering corresponds to that of table 1).

(a) Continuous rimming flows (see figure 2) were examined by Moffatt (1977) using the leading-order equation (2.8). As $M \rightarrow M_{*} \approx 4.44$, then $q \rightarrow \frac{2}{3}$ and the solution develops a 'corner' at $\theta=0$.

(b) Flows with shocks (see figure 3a) were examined by Benjamin et al. (1993) and O'Brien \& Gath (1998), using the leading-order theory (which allows the solution to have discontinuities). As $M \rightarrow M_{* *} \approx 6.93$, the shock approaches the bottom of the cylinder $\left(\theta=\frac{3}{2} \pi\right)$, while its amplitude grows.

(c) Near-critical rimming flows are examined in $\S 6$ of this paper. They can be interpreted as shock solutions with the shock being close to the bottom of the cylinder, modified by surface tension. As the amplitude of the solution grows, its peak 

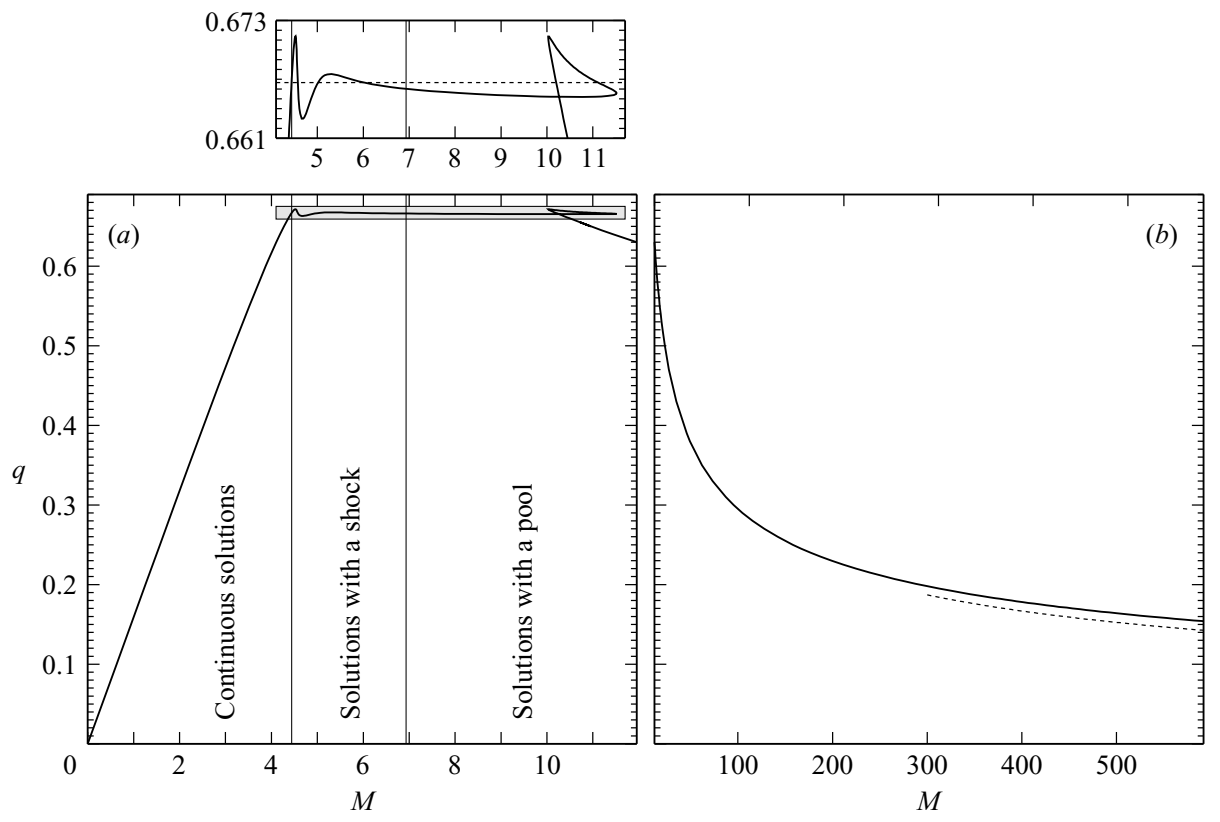

FIgURE 14. The flux $q$ vs. the net mass $M$, for $\epsilon=10^{-3}$. The numerical solution is shown as a solid line, the asymptotic solution (7.11) is shown as dotted line in (b). A blow-up of the region shaded in $(a)$ is shown above it.

(the 'pool') changes shape: initially, it is skewed to the left (which shows its relation to shocks); but, for larger amplitudes, the pool becomes symmetric (see figure 13). From a mathematical viewpoint, this regime, as well as the next one, are unusual, as they involve a variable number of asymptotic zones, depending on the small parameter $\epsilon$.

(d) Supercritical rimming flows are examined in $\S 3$ of this paper. This regime can be treated as a limiting case of near-critical flows (in particular, it has a symmetric pool). It can be demonstrated that, even though this regime was studied using the scaling $h_{\max }=O\left(\epsilon^{-1 / 5}\right)$, the results obtained are applicable to all cases with $h_{\max } \ll \epsilon^{-1 / 3}$.

(e) Strongly supercritical flows, i.e. such that $h_{\max } \gg \epsilon^{-1 / 3}$ were considered by Ashmore et al. (2003) and in $\S 7$ of this paper. The main characteristic feature of this regime is the small thickness $h_{\text {out }}$ of the film outside the pool and small flux $q$ (for all other regimes with shocks and pools, $h_{\text {out }}=O(1), q \approx \frac{2}{3}$ ). Mathematically, this case is much simpler than its two predecessors $(c)$ and $(d)$, as it involves only four asymptotic zones.

Observe that our classification has a 'gap', namely the regime with

$$
M=O\left(\epsilon^{-1 / 6}\right), \quad h_{\max }=O\left(\epsilon^{-1 / 3}\right),
$$

which describes the transition from the thick-outer-film regime $(d)$ to the thin-outerfilm regime $(e)$.

The regimes introduced above are best illustrated by plotting the flux $q$ as a function of the net mass $M$ - see figure 14. One can see that the intermediate regime (8.1) is an interesting one, as it allows for up to three solutions with the same net mass (see the three 'branches' which exist in the interval $10 \lesssim M \lesssim 11.5$ in figure $14 a$ ). The lower branch is an extension of the small- $q$ solution considered by Ashmore et al. (2003) and, thus, corresponds to a non-limiting outer solution. The middle and upper 

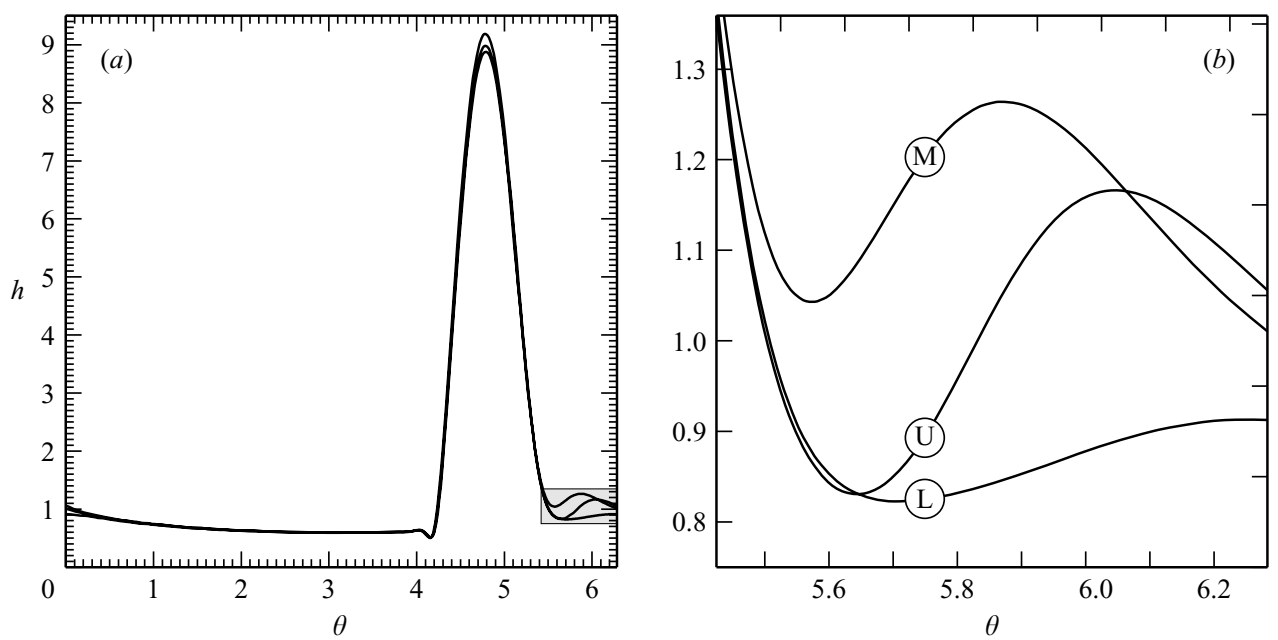

FIGURE 15. Examples of steady rimming flows (numerical solution) with the same net mass $(M=10.5)$ but different fluxes $q$, corresponding to the three 'branches' shown in figure 14 (in all three cases, $\epsilon=10^{-3}$ ). (b) A blow-up of the region shaded in $(a)$. Curve L corresponds to $q \approx 0.6598$ (lower branch); curve $\mathrm{M}$, to $q \approx 0.6652$ (middle branch); and curve $\mathrm{U}$, to $q \approx 0.6686$ (upper branch).

branches both involve the limiting outer solution and differ only in the right-hand transitional region (see figure 15). Note that figure 14 also validates the assumptions about the flux $q$ on which the present paper and Ashmore et al. (2003) are based: that $q \approx \frac{2}{3}$ for the near- and supercritical regimes and $q \ll 1$ for the strongly supercritical one.

Finally, simulations of the evolution equation (2.4) indicate that, for all regimes up to and including the supercritical regime, the solution always converges to the steady state with the corresponding value of $M$, which implies stability. What happens in further regimes is unclear, as the straightforward numerical methods are either too slow or too inaccurate, and this question requires further investigation.

\section{Appendix A. The streamlines of a rimming flow}

Let us introduce the non-dimensional radial and azimuthal velocities,

$$
u=\frac{u_{*}}{\alpha R \Omega}, \quad v=\frac{v_{*}}{R \Omega},
$$

and, also, the non-dimensional depth of the film

$$
z=\frac{R-r_{*}}{\alpha R}
$$

where $\alpha$ is given by (2.2) and asterisks, as before, mark dimensional variables. Within the framework of the lubrication approximation, $u$ and $v$ are given by (e.g. Benilov \& O’Brien 2005)

$$
\begin{gathered}
u=-\left(\frac{z^{3}}{6}-\frac{h z^{2}}{2}\right)\left[\sin \theta+\epsilon\left(\frac{\mathrm{d}^{2} h}{\mathrm{~d} \theta^{2}}+\frac{\mathrm{d}^{4} h}{\mathrm{~d} \theta^{4}}\right)\right]-\frac{h_{\theta} z^{2}}{2}\left[\cos \theta-\epsilon\left(\frac{\mathrm{d} h}{\mathrm{~d} \theta}+\frac{\mathrm{d}^{3} h}{\mathrm{~d} \theta^{3}}\right)\right], \\
v=1+\left(\frac{z^{2}}{2}-h z\right)\left[\cos \theta-\epsilon\left(\frac{\mathrm{d} h}{\mathrm{~d} \theta}+\frac{\mathrm{d}^{3} h}{\mathrm{~d} \theta^{3}}\right)\right]
\end{gathered}
$$


It can be verified by inspection that (A 1)-(A 2) correspond to the following streamfunction:

$$
\psi=z+\left(\frac{z^{3}}{6}-\frac{h z^{2}}{2}\right)\left[\cos \theta-\epsilon\left(\frac{\mathrm{d} h}{\mathrm{~d} \theta}+\frac{\mathrm{d}^{3} h}{\mathrm{~d} \theta^{3}}\right)\right] .
$$

Now, the streamlines can be obtained by equating $\psi$ to a constant,

$$
\psi(z, \theta)=\text { const. }
$$

For a given $h(\theta)$, (A 3)-(A 4) constitute a cubic equation for $z$ as a function of $\theta$ (obviously, only those roots should be taken for which $\operatorname{Im} z=0,0 \leqslant \operatorname{Re} z \leqslant h$ ).

\section{Appendix B. Solution of problem (7.8)-(7.10)}

Let us rewrite (7.8)-(7.10) in terms of

$$
\xi=\frac{x_{1}}{\hat{q}}, \quad \eta=\frac{h_{1}}{\hat{q}},
$$

which yields

$$
\begin{aligned}
& \eta \rightarrow 1 \quad \text { as } \quad \xi \rightarrow+\infty \text {, } \\
& \eta \rightarrow \hat{q}\left(\frac{2}{3}\right)^{1 / 2} \xi^{2} \quad \text { as } \quad \xi \rightarrow-\infty \text {. } \\
& \eta+\frac{1}{3} \eta^{3} \frac{\mathrm{d}^{3} \eta}{\mathrm{d} \xi^{3}}=1 .
\end{aligned}
$$

To find asymptotics of $\eta$ as $x \rightarrow+\infty$, seek a solution in the form

$$
\eta=1+\tilde{\eta}(x),
$$

substitute (B 4) in equation (B 3), linearize it,

$$
\tilde{\eta}+\frac{\mathrm{d}^{3} \tilde{\eta}}{\mathrm{d} \xi^{3}}=0
$$

and solve:

$$
\tilde{\eta}=\exp \left(\frac{1}{2} \xi\right)\left[c_{1} \sin \left(\frac{3^{1 / 2} \xi}{2}\right)+c_{2} \cos \left(\frac{3^{1 / 2} \xi}{2}\right)\right]+c_{3} \exp (-\xi),
$$

where $c_{1}, c_{2}, c_{3}$ are constants of integration. Observe that (B 4)-(B 5) satisfy the boundary condition (B 1) only if $c_{1}=c_{2}=0$; note also that (B 1)-(B 3) are invariant with respect to the transformation $\xi \rightarrow \xi+$ real constant, and we can assume real constant $=\ln \left|c_{3}\right|$ which yields either

$$
\eta=1+\exp (-\xi) \quad \text { as } \quad \xi \rightarrow+\infty
$$

or

$$
\eta=1-\exp (-\xi) \quad \text { as } \quad \xi \rightarrow+\infty .
$$

Using (B 6) or (B 7), we can 'shoot' the solution (numerically) from a large positive value of $\xi$ towards $-\infty$. Once the solution's second derivative becomes close to a constant (hence the boundary condition (B 2) is almost satisfied), we can 'collect' the value of $\hat{q}$ from

$$
\hat{q}=\frac{1}{2}\left(\frac{3}{2}\right)^{1 / 2} \lim _{\xi \rightarrow-\infty} \frac{\mathrm{d}^{2} \eta}{\mathrm{d} \xi^{2}} .
$$


It turns out that the boundary condition (B 7) does not yield a solution with the required (parabolic) asymptotics for $\xi \rightarrow-\infty$, as $\eta(\xi)$ passes through zero at a finite value of $\xi$ and becomes negative. Condition (B 6), in turn, yields the required asymptotics, with $\hat{q} \approx 0.82$.

\section{REFERENCES}

Ashmore, J., Hosoi, A. E. \& Stone, H. A. 2003 The effect of surface tension on rimming flows in a partially filled rotating cylinder. J. Fluid Mech. 479, 65-98.

Benilov, E. S. \& O’Brien, S. B. G. 2005 Inertial instability of a liquid film inside a rotating horizontal cylinder. Phys. Fluids 17, 052106.

Benjamin, T. B., Pritchard, W. G. \& Tavener, S. J. 1993 Steady and unsteady flows of a highly viscous liquid inside a rotating horizontal cylinder. Unpublished manuscript.

Hinch, E. J. 1991 Perturbation Methods. Cambridge University Press.

JeONG, J.-T. \& Moffatt, H. K. 1992 Free-surface cusps associated with a flow at low Reynolds number. J. Fluid Mech. 241, 1-22.

Johnson, R. E. 1990 Coating flow stability in rotating molding. In Engineering Science, Fluid Dynamics: A Symposium to Honor T.Y. Wu (ed. G. T. Yates), pp. 435-449. World Scientific.

Landau, L. \& Levich, B. 1942 Dragging of liquid by a plate. Acta Physiochim. USSR 17, 42-54.

Moffatt, H. K. 1977 Behaviour of a viscous film on the outer surface of a rotating cylinder. $J$. Fluid Mech. 16, 651-574.

O'Brien, S. B. G. 2002 Linear stability of rimming flows. Q. Appl. Maths. 60, 201-212.

O'Brien, S. B. G. \& Gath, E. G. 1998 The location of a shock in rimming flow. Phys. Fluids 10, $1040-1042$.

Press, W. H., Teulkolsky, S. A., Vetterling, W. T. \& Flannery, B. P. 1992 Numerical Recipes, 2nd Edn. Cambridge University Press.

Wilson, S. D. R. 1982 The drag-out problem in film coating theory. J. Engng Maths 16, 209-221.

Wilson, S. D. R. \& JonES, A. F. 1983 The entry of a falling film into a pool and the air-entrainment problem. J. Fluid Mech. 128, 219-230. 